This item was submitted to Loughborough's Research Repository by the author.

Items in Figshare are protected by copyright, with all rights reserved, unless otherwise indicated.

\title{
Depth-resolved full-field measurement of corneal deformation by optical coherence tomography and digital volume correlation
}

\section{PLEASE CITE THE PUBLISHED VERSION}

http://dx.doi.org/10.1007/s11340-016-0165-y

\section{PUBLISHER}

Springer (๑ Society for Experimental Mechanics)

\section{VERSION}

AM (Accepted Manuscript)

\section{PUBLISHER STATEMENT}

This work is made available according to the conditions of the Creative Commons Attribution-NonCommercialNoDerivatives 4.0 International (CC BY-NC-ND 4.0) licence. Full details of this licence are available at: https://creativecommons.org/licenses/by-nc-nd/4.0/

\section{LICENCE}

CC BY-NC-ND 4.0

\section{REPOSITORY RECORD}

Fu, Jiawei, Maryam Haghighi Abyaneh, Fabrice Pierron, and Pablo Ruiz. 2016. "Depth-resolved Full-field Measurement of Corneal Deformation by Optical Coherence Tomography and Digital Volume Correlation". Loughborough University. https://hdl.handle.net/2134/21022. 


\title{
Depth-resolved Full-Field Measurement of Corneal Deformation by Optical Coherence Tomography and Digital Volume Correlation
}

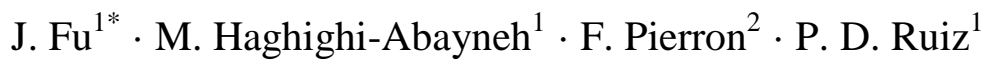

\begin{abstract}
The study of vertebrate eye cornea is an interdisciplinary subject and the research on its mechanical properties has significant importance in ophthalmology. The measurement of depth-resolved 3D full-field deformation behaviour of cornea under changing intraocular pressure is a useful method to study the local corneal mechanical properties. In this work, optical coherence tomography was adopted to reconstruct the internal structure of a porcine cornea inflated from $15 \mathrm{mmHg}$ to $18.75 \mathrm{mmHg}$ (close to the physical porcine intraocular pressure) in the form of 3D image sequences. An effective method has been developed to correct the commonly seen refraction induced distortions in the optical coherence tomography reconstructions, based on Fermat's principle. The 3D deformation field was then determined by performing digital volume correlation on these corrected 3D reconstructions. A simple finite element model of the inflation test was developed and the predicted values were compared against digital volume correlation results, showing good overall agreement.
\end{abstract}

Keywords Cornea $\cdot$ Optical coherence tomography $\cdot$ Refraction correction $\cdot$ Digital volume correlation $\cdot 3 D$ fullfield deformation measurement $\cdot$ Inflation test

\footnotetext{
${ }^{1}$ Wolfson School of Mechanical and Manufacturing Engineering, Loughborough University, Loughborough, LE11

3TU, United Kingdom

${ }^{2}$ Faculty of Engineering and the Environment, University of Southampton, Southampton, SO17 1BJ, United

Kingdom

*Corresponding author

Tel: +44 (0)2380 592891

Fax: +44 (0)2380 593190

E-mail: jiawei.fu@alumni.lboro.ac.uk
} 


\section{Introduction}

The vertebrate eye cornea plays an important role as the main refractive element for image formation, provides a protective envelope for the internal ocular elements and maintains the shape of the eye [1, 2]. Its structural integrity, shape and transparency are essential to maintain optical performance. Abnormalities in its composition or structure due to disorders or disease such as keratoconus, corneal dystrophy, glaucoma or mass lesions, to name a few, severely degrade vision [3-7]. Ablative laser surgery is widely used to correct refractive performance and improve visual acuity, with more than one million operations in the United States alone each year [8]. Corneal thickness, rather than corneal mechanical response, is one of the main criteria used during screening, to decide the suitability of a particular cornea to ablative surgery. Due to the hydrostatic intraocular pressure, changes in the local stiffness due to ablation can result in residual refractive errors as the final corneal profile does not correspond to the target profile aimed by the surgeon. For this reason, the corneal mechanical response, rather than thickness alone, is considered as a more suitable screening criterion. Quantification of mechanical response, when done through predictive models, e.g. based on finite elements, requires as input the corneal geometry, loads, boundary conditions and stiffness parameters which may vary through the thickness [9-12].

So far, most of the research work that has been done towards identification of corneal mechanical properties was based on point-wise or surface deformation measurements in corneal inflation tests using stereoscopic camera systems and the surface deformation measurement technique digital image correlation (DIC) [13-16]. Many researchers also use tonometers to study the biomechanical properties of cornea and their relationship to intraocular press (IOP), the fluid pressure inside the eye. Using tonometers IOP can be measured either in a contact way such as Goldmann applanation tonometer (GAT) and Pascal dynamic contour tonometer (PDCT) [17-19] or, more recently, in a noncontact way through using an air impulse such as ocular response analyzer (ORA) [20, 21] and Corvis tonometer [22-24].

Optical coherence tomography (OCT) is a non-invasive, non-contacting imaging technique that can acquire micrometer resolution, cross sectional images from within semi-transparent, light scattering media such as biological tissues. It is based on low coherence interferometry through the use of broadband light sources that can emit light over a broad range of frequencies [25-28]. OCT elastography (OCE) has been used for mapping strain and elastic modulus in soft tissues [29-33]. Generally, it determines the deformation in the OCT image sequences (B-scans) by applying 2D cross-correlation algorithms (CCOCE) to calculate speckle motion due to applied forces [30, 33, 34]. 
Alternatively, the deformation can be determined by measuring the phase change between successive A-scans or Bscans due to tissue motion, which is called phase-sensitive OCT elastography (PSOCE) [29, 32, 35]. Researchers [36-38] have combined phase-sensitive OCE and air-puff devices or other actuators [39, 40] to measure the amplitude and speed of the stimulated elastic wave, from which the biomechanical properties of corneal tissues such as corneal elasticity and viscoelasticity can be characterized, both in vitro and in vivo. OCE is a useful method to quantify the elastic behaviour of soft tissues. However, most of the applications have so far focused on the measurements with only axial sensitivity, especially for PSOCE. For a more comprehensive study of the material behaviour (e.g. deformation in anisotropic material) as well as the prerequisite for the identification of 3D constitutive parameters using inversion methods such as finite element model updating (FEMU) [41-43], the virtual fields method (VFM) [44-47] or the constitutive compatibility method (CCM) [48], measurement of the depthresolved 3D deformation is required to avoid simplistic assumptions of isotropy or depth independent properties. This can be implemented by either 1) using a system with sensitivity to all deformation components based on a wavelength scanning OCT system with multiple illumination directions as introduced by Chakraborty and Ruiz [49] or 2) using a single channel OCT system to reconstruct the corneal internal structure, followed by digital volume correlation (DVC) to provide deformation fields with all displacement components.

DVC is the 3-D extension of the widely applied DIC used to measure surface deformations. It was developed to obtain the bulk deformation behaviour of materials by tracking internal features that resemble 3D speckle patterns contained in the reconstructed volumes and was introduced by Bay, Smith [50]. DVC is becoming a popular 3D deformation measurement technique, especially applied on X-ray computed tomography (X-ray CT) reconstructed volumes of materials such as metals, composites, foams and hard tissues like trabecular bone [51-54]. For these materials, a volume of pattern contrast is provided by X-ray CT based on the differences in X-ray absorption of the different material constituents. For soft semi-transparent materials such as biological tissues that do not offer good contrast for $\mathrm{X}$ rays, OCT is currently the most suitable technique for 3D reconstruction. However, due to the corneal curvature, refraction distortion introduces localization errors in internal features. These have to be accounted for in order to properly map displacement and strain fields through-the-thickness. Moreover, the effect of the refractive index must be taken into account to re-map the corneal volume reconstruction in a coordinate system that represents depth, rather than optical path difference in the axial direction. As far as the present authors are concerned, only one study has been published on the application of OCT and DVC to cornea [55]. However, this was done on a small flat 
sample cut from a porcine cornea and loaded in uniaxial compression, providing a much simpler configuration for which the refraction problem did not occur. The present study aims at performing such measurements on a full cornea submitted to an inflation test.

The current study presents a methodology to measure all the components of the displacement field inside the volume of a cornea using OCT and DVC. These results are necessary to identify the corneal elastic stiffness parameters as in previous work [45]. Section 2 presents the materials and methods used to evaluate 3D corneal displacement fields in an inflation test, including sample preparation, OCT system, refraction correction and its performance and DVC displacement evaluation. In Section 3 we examine the experimental results and discuss the main advantages and limitations of the proposed methodology.

\section{Materials and Methods}

\subsection{Materials}

Porcine corneas were used in this study. A fresh ocular globe was collected from a local abattoir within 6 hours after slaughter and the corneas were tested within 12 hours post-mortem. The ocular globes were stored in a refrigerator below $4^{\circ} \mathrm{C}$ to prevent tissue degeneration. It was then brought to room temperature half an hour before testing to ensure thermal equilibrium with the environment. A corneal trephinate (circular cut-off that includes the cornea and a $2 \mathrm{~mm}$ sclera ring, shown in Fig. 1 from different views), was excised from the corneal globe and stored in saline solution $0.9 \% \mathrm{NaCl}$. The central corneal thickness was measured using a direct computer control (DCC) coordinate measuring machine. During the measurement, the coordinates of the apex of a corneal shaped support were first recorded when the touch-point sensor made contact with it. The corneal trephinate was then mounted on the support with its apex over the apex of the support. The coordinates of the corneal apex were recorded and the thickness of the cornea determined by evaluating the axial distance between the two measured points. The corneal trephinate thickness was $1.53 \pm 0.03 \mathrm{~mm}$, which includes a slight swelling due to water absorption in the stroma. 

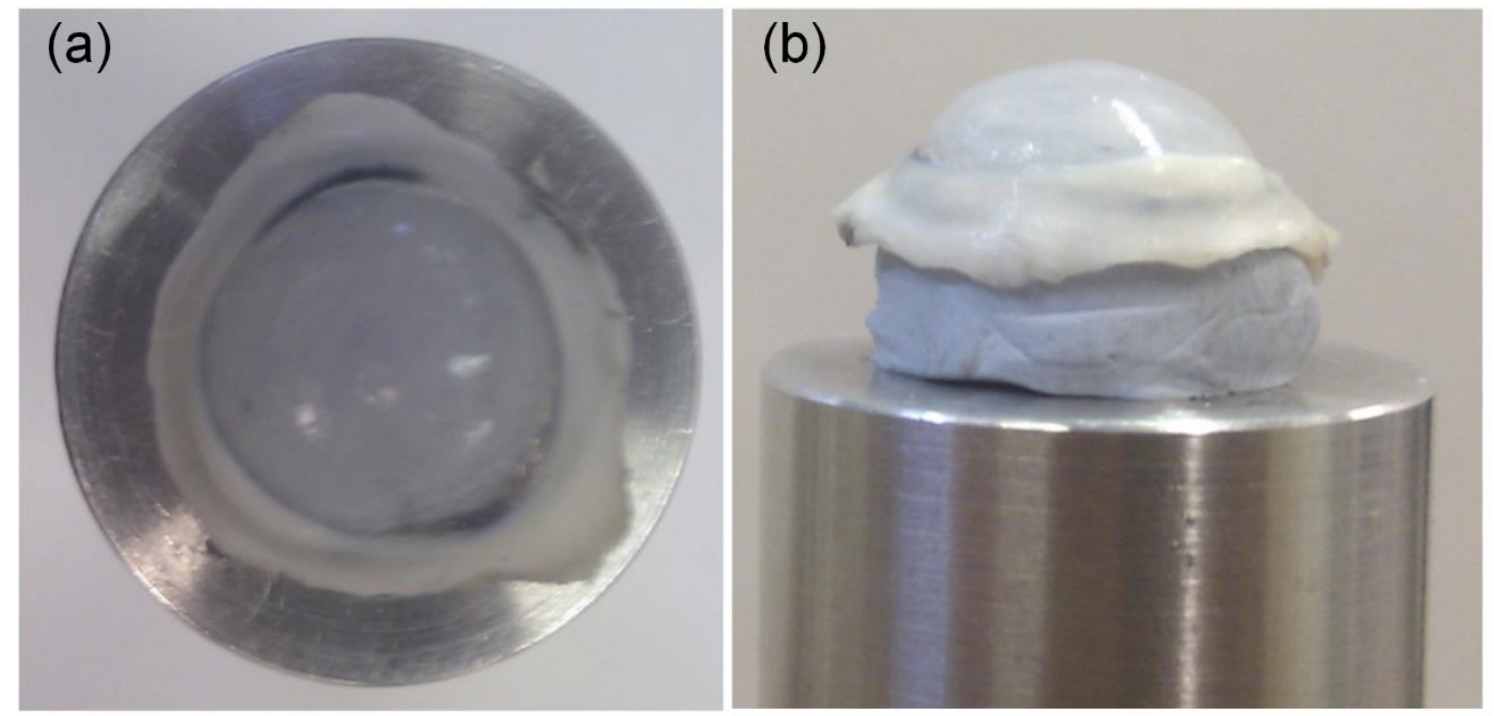

Fig 1. Porcine corneal trephinate from different views.

\subsection{Experimental set-up and image acquisition}

The experimental set-up is illustrated in Fig. 2, which consists of an inflation test cell and the SS-OCT system (Thorlabs OCS1300SS, central wavelength $1325 \mathrm{~nm}$, spectral bandwidth $100 \mathrm{~nm}$, lateral resolution $25 \mu \mathrm{m}$, depth resolution $12 \mu \mathrm{m}$ in air, axial scan rate $32 \mathrm{kHz}$ ). The sample was mounted and fixed on an artificial anterior chamber (AAC) by a locking ring. This chamber has inlet and outlet ports for the fluid to adjust the internal pressure and another port connected to a pressure transducer. The chamber pressure was controlled by adjusting a $1 \mathrm{ml}$ microsyringe connected to the inlet port. The porcine corneal trephinate was first inflated to $15 \mathrm{mmHg}$. Under this pressure state the sample maintained a smooth and taut anterior surface; this state served as the reference configuration. It was then inflated to $18.75 \mathrm{mmHg}$, which is close to the physical porcine intraocular pressure to study the corneal deformation under the pressure change. The test was carried out at room temperature. 

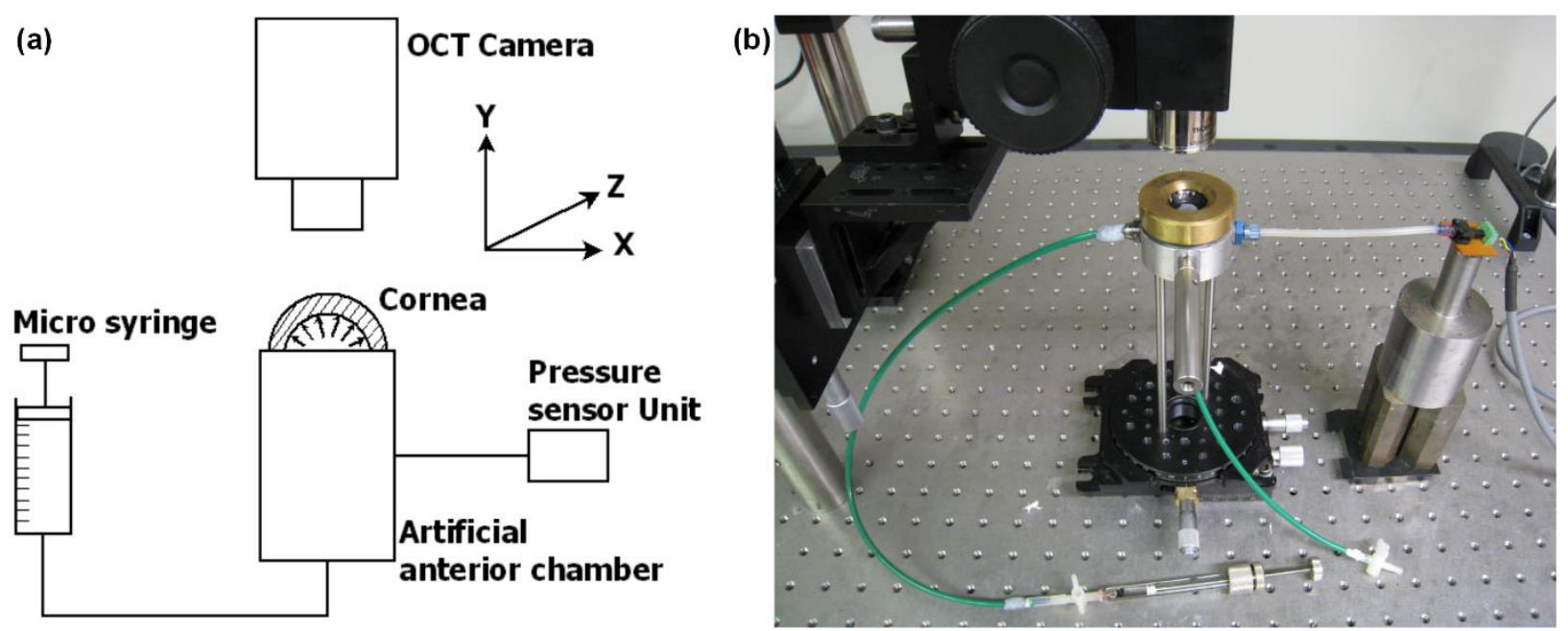

Fig 2. (a) Schematic diagram, and (b) experimental set-up.

At each pressure state, a 3D volume data cube of the cornea sample was acquired using the SS-OCT system. The system uses a rapidly tuned narrowband light source. During image acquisition, a scanning beam is first split into two arms, the sample arm and the reference arm. The light back-scattered from the sample arm interferes with that from the reference arm and the frequency of the interference signal is proportional to their optical path difference. A single photodetector is used to record the interference signal. Based on the Fourier transform of the interference signal of each wavelength scan, depth profiles (A-scans) of the test sample can be obtained with a maximum depth range of approximately $3 \mathrm{~mm}$. By performing adjacent A-scans, an image in the $x y$ plane is created. Combing the multiple $2 \mathrm{D}$ image sequence in the $z$-direction, the 3D volume of the test sample is reconstructed. In the present study, the dimensions of the reconstructed volumes are $1024 \times 512 \times 1024$ voxels, corresponding to a $11 \times 3 \times 11 \mathrm{~mm}^{3}$ region of interest along the $x, y$ and $z$ directions, respectively. For each 3-D volume the acquisition time is around 5 minutes. The reconstructed volumes for the reference and deformed states were recorded to compute the depth-resolved 3D full-field deformation by performing DVC. In Fig. 3(a) and 3(b) a quarter of the reconstructed volume and a typical central $z$-slice (cut in $x y$-plane at the coordinate of $z, 1024 \times 512$ voxels) of the porcine cornea sample are illustrated. For the OCT reconstructions, the voxel sizes along the lateral scanning directions $(x$ and $z$ ) and the vertical optical path direction (through thickness, $y$ ) are not identical since the latter depends on the refractive index of the medium. Here, along the lateral $x$ and $z$ directions, the voxel size is $10.7 \mu \mathrm{m}$, determined by dividing the $11 \mathrm{~mm}$ side length of the field of view by the number of corresponding voxels, 1024 . 
Along the y direction, the voxel size within the porcine corneal trephinate is $4.5 \mu \mathrm{m}$, determined by dividing its thickness, $1.53 \mathrm{~mm}$, by the number of corresponding voxels, 340 .
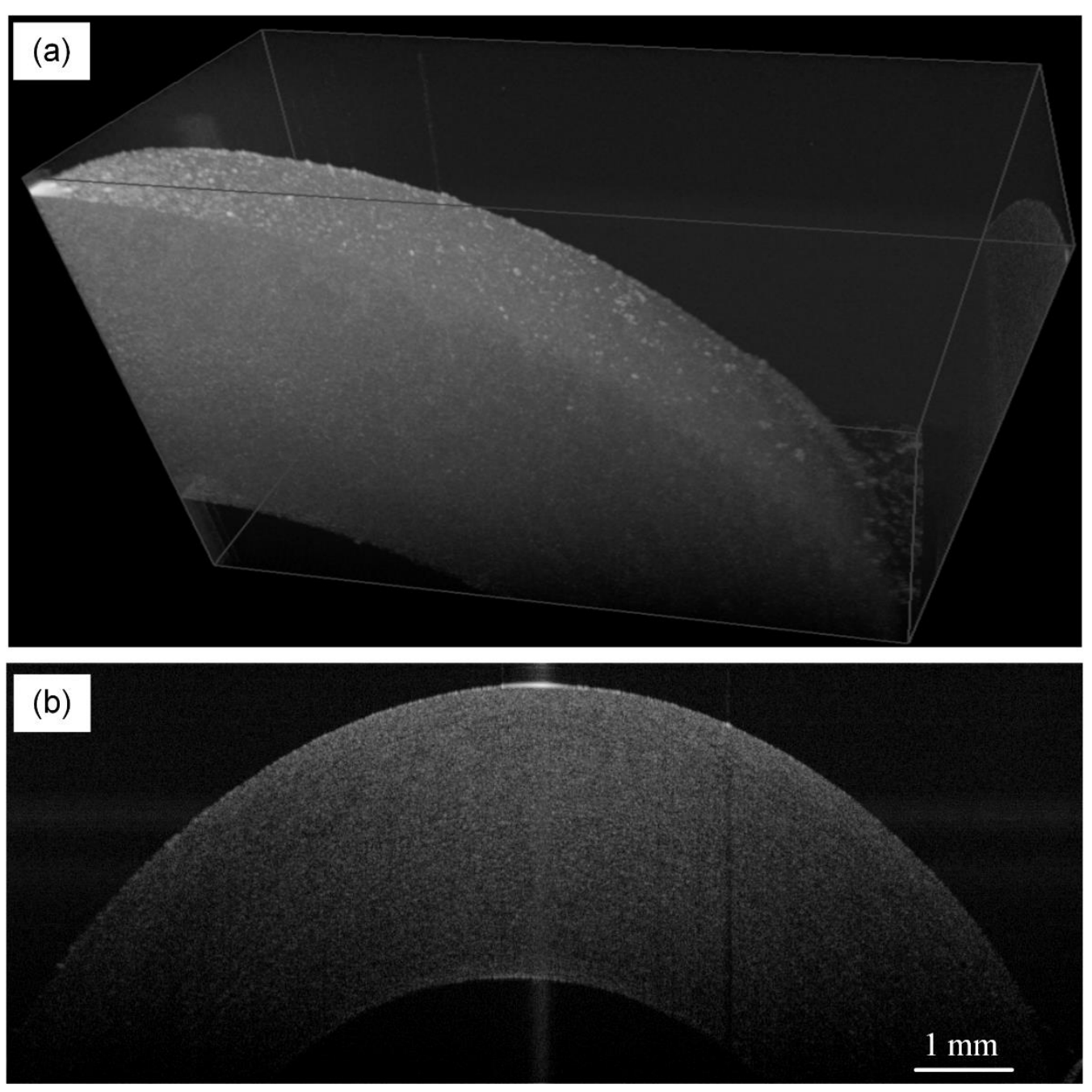

Fig 3. (a) Part of the reconstructed porcine corneal trephinate volume in 3D view, and (b) central z-slice, obtained with the SS-OCT system.

\subsection{Correction of refraction induced distortion in OCT reconstructions}

OCT reconstructions usually suffer from different types of geometric distortions. Refraction induced distortion arises when the sample has a curved surface or the sample has a flat surface which does not lie perpendicular to the scanning beam. In these cases the scanning beam changes its propagation direction at the air/sample interface but the 
OCT system reconstructs the scattered light as if light had propagated without such a change in direction. A consequence of this is that internal features appear to be in a different position in the OCT reconstruction. Moreover, as the dimension along the propagation direction in an OCT image is optical path rather than physical distance, the position of features depends on the knowledge of the refractive index of the medium. Another type of distortion is caused by the 'fan' scanning of certain OCT systems, in which a fan of scanning rays cause a flat surface to appear curved in the OCT reconstruction [56-58]. The SS-OCT system used in this work was designed to eliminate fan distortion, and therefore flat surfaces are reconstructed as flat in the OCT data volume. This is done by placing the galvo-mirrors in the back focal plane of the objective lens so that the beam pivots about it. In this work, the distortion correction needs only to consider the refraction at the air/cornea interface, given that no fan distortion is present. A uniform distribution of refractive index will be assumed within the stroma considering its very small local variations, e.g. 1.373-1.380 as reported by Patel, Marshall [59].

Figure 4 illustrates how refraction distortion arises. Let $K$ represent the plane of zero optical path difference (a datum surface relative to which distance is measured). $A$ is an arbitrary incident point on the top surface at which the scanning beam, travelling perpendicular to $K$, enters the cornea. $A C$ is the normal to the surface at the incident point $A$. The incident ray $O A$ makes an angle $\theta_{0}$ to the surface normal at $A$. The refracted ray passes through point $B$ within the corneal tissue (object space), making an angle $\theta_{1}$ to the surface normal at $A$, following Snell's law,

$$
n_{0} \sin \theta_{0}=n_{1} \sin \theta_{1}
$$

where $n_{0}$ and $n_{1}$ represent the refractive indices of the surrounding air and the cornea, taken as 1 and 1.38 , respectively. The OCT system records this refracted optical path in the image as a vertical A-scan line, denoted $O I$ in Fig. 4, where point $I$ is the corresponding point of object point $B$ in the reconstructed image space. As the optical path difference is equal to the physical distance in the medium multiplied by the refractive index, $O I$ in the image space is given by

$$
|O I|=n_{0}|O A|+n_{1}|A B|
$$

where || denotes Cartesian distance. It can be seen that a point $I$ in the image space actually corresponds to point $B$ in the object space. Therefore, the reconstructed volume appears as distorted with respect to the real configuration of the object. The distorted data volume would then introduce errors in the deformation measurements obtained by performing DVC on the OCT reconstructions. 
Refraction correction in 2D OCT images has already been dealt with in the literature using Fermat's principle and Snell's law $[57,60]$. The approach based on Fermat's principle does not require any assumptions on the cornea surface shape (e.g. approximation to a sphere). This principle states that the path taken between two points by a ray of light is the path that can be reached in the least time. Thus, point $B$ is reached by a unique ray refracted at point $A$. Therefore, given $B$ and knowing that the incidence direction is perpendicular to $K$, point $A$ can be found by minimizing the optical path defined in Eqn. (2). Then, the corresponding point $I$ in the image space (to point $B$ in object space) can be searched along path $O A$ at a distance $n_{1}|A B|$ from point $A$. Once the non-integer coordinates of point $I$ are determined, the intensity value at this image point is evaluated through interpolation (trilinear in our case), and the value is associated to the coordinates of point $B$, which belong to a Cartesian grid in object space. By applying the same procedure to all other object points, the corrected volume is obtained.

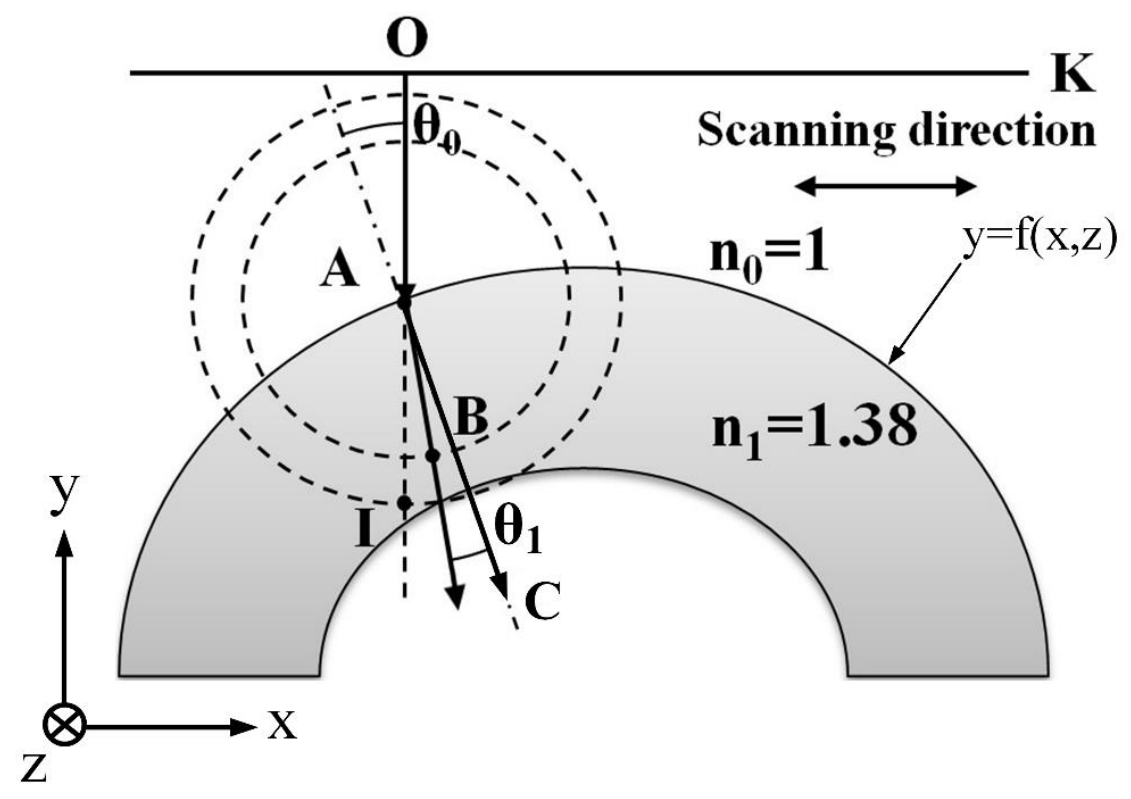

Fig 4. Refraction of the incident beam in an OCT scanning system. During a scan, the beam moves parallel to the indicated direction along the $\mathrm{x}$ and $\mathrm{z}$ axes.

The refractive indices of the media and a parametric description of the interface must be first established. In Fig. 4, the top interface of the object can be described by a surface

$$
y=f(x, z)
$$


where $y=0$ corresponds to the datum plane $K$. It should be pointed out that the DC term in $f(x, z)$ is not critical as refraction only depends on the local gradient. An arbitrary object point $B$ with coordinates $\left(x_{B}, y_{B}, z_{B}\right)$ has an associated entry point $A$ with coordinates $\left(x_{A}, f\left(x_{A}, z_{A}\right), z_{A}\right)$. From Eqn. (2), the optical path $|O B|$ can be written as

$$
|O B|=n_{0} f\left(x_{A}, z_{A}\right)+n_{1}\left[\left(x_{B}-x_{A}\right)^{2}+\left(y_{B}-f\left(x_{A}, z_{A}\right)\right)^{2}+\left(z_{B}-z_{A}\right)^{2}\right]^{\frac{1}{2}}
$$

where $\left(x_{B}, y_{B}, z_{B}\right)$ is known as it is defined in a 3-D Cartesian grid in object space for each point. The only unknowns in Eqn. (4) are $x_{A}$ and $z_{A}$ as $f(x, z)$ is determined from the OCT reconstruction of the top surface. In this case, $|O B|$ becomes a function of $x_{A}$ and $z_{A}$, which avoiding the index $A$ writes

$$
|O B|(x, z)=n_{0} f(x, z)+n_{1}\left[\left(x_{B}-x\right)^{2}+\left(y_{B}-f(x, z)\right)^{2}+\left(z_{B}-z\right)^{2}\right]^{\frac{1}{2}}
$$

By minimizing $|O B|, x_{A}$ and $z_{A}$ can be found. Then, the coordinates of $A$ are found at $\left(x_{A}, f\left(x_{A}, z_{A}\right), z_{A}\right)$. The next task is to determine the coordinates $\left(x_{I}^{\prime}, y_{I}^{\prime}, z_{I}^{\prime}\right)$ of point $I$ in the image space that represents point $B$ in the object space. Since the OCT system registers the optical path along a vertical A-scan, the horizontal coordinates $x_{I}^{\prime}$ and $z_{I}^{\prime}$ of point $I$ are

$$
\begin{aligned}
& x_{I}^{\prime}=x_{A} \\
& z_{I}^{\prime}=z_{A}
\end{aligned}
$$

while the vertical coordinate $y_{I}^{\prime}$ is

$$
y_{I}^{\prime}=n_{0} f\left(x_{A}, z_{A}\right)+n_{1}|A B|
$$

where $|A B|$ is

$$
|A B|=\left[\left(x_{B}-x_{A}\right)^{2}+\left(y_{B}-f\left(x_{A}, z_{A}\right)\right)^{2}+\left(z_{B}-z_{A}\right)^{2}\right]^{\frac{1}{2}}
$$

Once the coordinates of the image point $I$ are established, the grey level obtained through interpolation at that point will be associated to point $\mathrm{B}$ in the object space. The correction method was implemented in MatLab® R2007a using the Nelder-Mead simplex direct search algorithm to minimize $|O B|$. A parametric function $f(x, z)$ of the top interface was determined by picking points on the cornea/air interface from the OCT reconstruction and then fitting a fourth order polynomial. 
Figure 5 illustrates the central $z$-slice of the reconstructed corneal volume before and after the refraction correction. The vertical axis in the corrected image corresponds to spatial coordinates, rather than optical path as before correction.
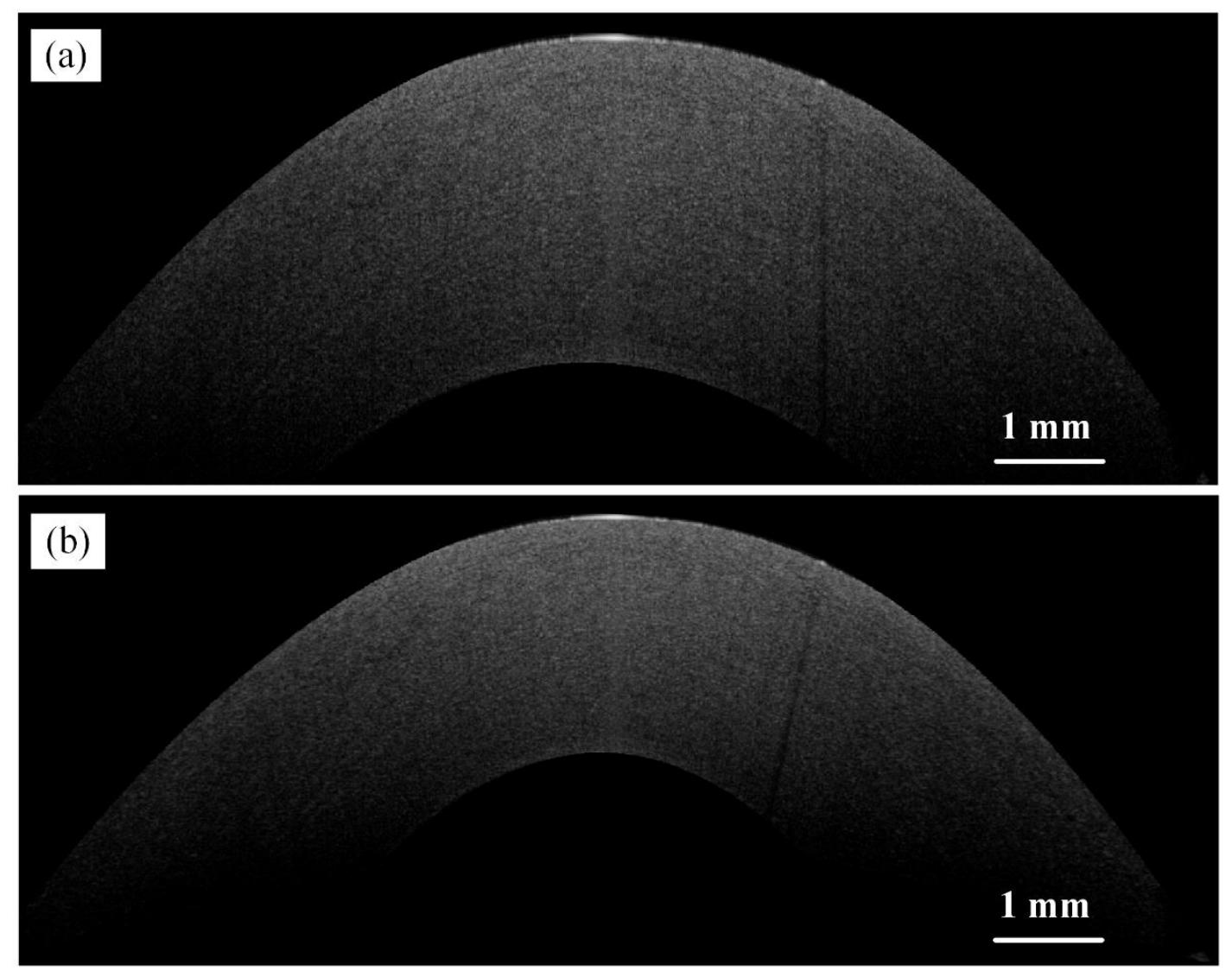

Fig 5. Central z-slice of the porcine cornea: (a) before and (b) after refraction correction

Alternative to the above Fermat's principle method, it is also possible to perform the refraction correction through a direct ray tracing method. In this case, the coordinate $x_{A}$ for any A-scan is known from the column location in the image. As shown in Fig. 4, assuming the parametric function of the top interface is known (fitted as for the Fermat's principle method), the incident angle $\theta_{0}$ for each scanning ray $O A$ can be determined after calculating the surface normal at incident point $A$, from which the refractive angle $\theta_{I}$ can be determined based on the Snell's law as defined in Eqn. (1). Then, the parametric function of the refractive ray $A B$ can be determined. For each image point $I$ with integer coordinates, the corresponding object point $B$ in the object space can be searched along the path $A B$ at a 
distance $|A I| / n_{1}$ from point $A$. Once the exact (non-integer) coordinates of point $B$ in the object space are determined, the light intensity value at the image point $I$ is associated to the coordinates of point $B$. Finally, since the corresponding object points are at non-integer coordinates, interpolation is needed to determine the light intensity value at the integer positions defined on a Cartesian grid in the object space. This approach is expected to reduce computation time. Work is currently underway to study the overall performance of the ray tracing method for a more efficient refraction correction.

\subsection{Digital volume correlation}

When performing DVC, the correlated volumes are first divided into sub-volumes defined on a 3D virtual grid. Each sub-volume contains a certain number of voxels (and material features or 3D speckles that will be tracked). The displacement vector of each sub-volume is calculated by tracking and matching the voxels of the sub-volumes in the

reference and deformed states. Assuming that $(x, y, z)$ and $(\hat{x}, \hat{y}, \hat{z})$ represent the coordinates of a given material point in the reference and deformed states, respectively, these coordinates are linked by the 3D affine transformation defined as a rigid body translation combined with displacement gradients. Thus,

$$
\begin{aligned}
& \hat{x}=x+U_{x}+\frac{\partial U_{x}}{\partial x} \Delta x+\frac{\partial U_{x}}{\partial y} \Delta y+\frac{\partial U_{x}}{\partial z} \Delta z \\
& \hat{y}=y+U_{y}+\frac{\partial U_{y}}{\partial x} \Delta x+\frac{\partial U_{y}}{\partial y} \Delta y+\frac{\partial U_{y}}{\partial z} \Delta z \\
& \hat{z}=z+U_{z}+\frac{\partial U_{z}}{\partial x} \Delta x+\frac{\partial U_{z}}{\partial y} \Delta y+\frac{\partial U_{z}}{\partial z} \Delta z
\end{aligned}
$$

where $U_{x}, U_{y}$, and $U_{z}$ are the rigid body translation of the sub-volume centre in the $x, y$ and $z$-direction, respectively. $\Delta x, \Delta y$ and $\Delta z$, represent the distance between the sub-volume centre and apoints of coordinates $(x, y, z)$ in the sub-volume. The local displacement gradients in Eqn. (9) characterize the rigid body rotation and the local stretch of the sub-volume. The displacement is obtained by maximizing a correlation coefficient that measures the degree of similarity of the gray-level distributions in the sub-volumes in both states. In the present work, this was accomplished using DaVis ${ }^{\circledR}$ (LaVision) software package based on a fast Fourier transform (FFT) algorithm. It evaluates the normalized cross correlation coefficient (NCC) defined as

$$
N C C=\frac{\sum \sum \sum F(x, y, z) G(\hat{x}, \hat{y}, \hat{z})}{\sqrt{\sum \sum \sum[F(x, y, z)]^{2} \sum \sum \sum[G(\hat{x}, \hat{y}, \hat{z})]^{2}}}
$$


where $F(x, y, z)$ and $G(\hat{x}, \hat{y}, \hat{z})$, respectively, stand for the grey levels at a material point $(x, y, z)$ in the reference state and the corresponding point $(\hat{x}, \hat{y}, \hat{z})$ in the deformed state. A double-pass approach was used whereby large sub-volumes were initially used to capture large displacements. Subsequent to this, these initial displacements were used to displace smaller sub-volumes, and thus ensure the pattern was followed and signal to noise ratio increased. Gaussian curve-fitting of the correlation function peak was used to find its position with sub-voxel resolution, which corresponds to the displacement. Trilinear interpolation was used for the sub-voxel interpolation. As the strain level in the present study is low, infinitesimal strain formulation can be used. Strains were obtained through centred finite difference of the displacement components, using the well-known relationship:

$$
\varepsilon_{i j}=\frac{1}{2}\left(U_{i, j}+U_{j, i}\right)
$$

where $U$ represents the displacement components along the $x, y$ and $z$-direction. Commas stand for the partial derivatives. No smoothing has been used prior to differentiation to maintain maximal spatial resolution as the strains were large enough compared to the noise floor estimated in the next section.

\subsection{Effect of the refraction correction on measurement performances}

To evaluate the performance of the refraction correction method and its ability to correct DVC results, rigid body rotation tests were carried out on two phantoms (silicone rubber MM240-TV seeded with copper particles with an average diameter of $1 \mu \mathrm{m}$ ). One is a flat strip while the other is a solid hemisphere. These two shapes were chosen as they enable us to study distortions induced by a uniform refraction angle (flat top surface) and non-uniform refraction angles (hemispherical top surface). As illustrated in Fig. 6, the phantoms were rotated by $0.5^{\circ}$ in the $x y$ plane. The dashed lines in the two OCT images illustrate the positions of these phantoms after rigid body rotation. DVC was performed to obtain the strain and rotation maps both before and after refraction correction. These results are compared to the reference values ( 0 strain and $0.5^{\circ}$ rotation) to check the effects of the refraction correction on the DVC measurements.

The strains were derived from the same centred finite difference of the displacement fields as in Eqn. (11) and the rotations were derived from

$$
\omega_{i j}=\frac{1}{2}\left(U_{i, j}-U_{j, i}\right)
$$



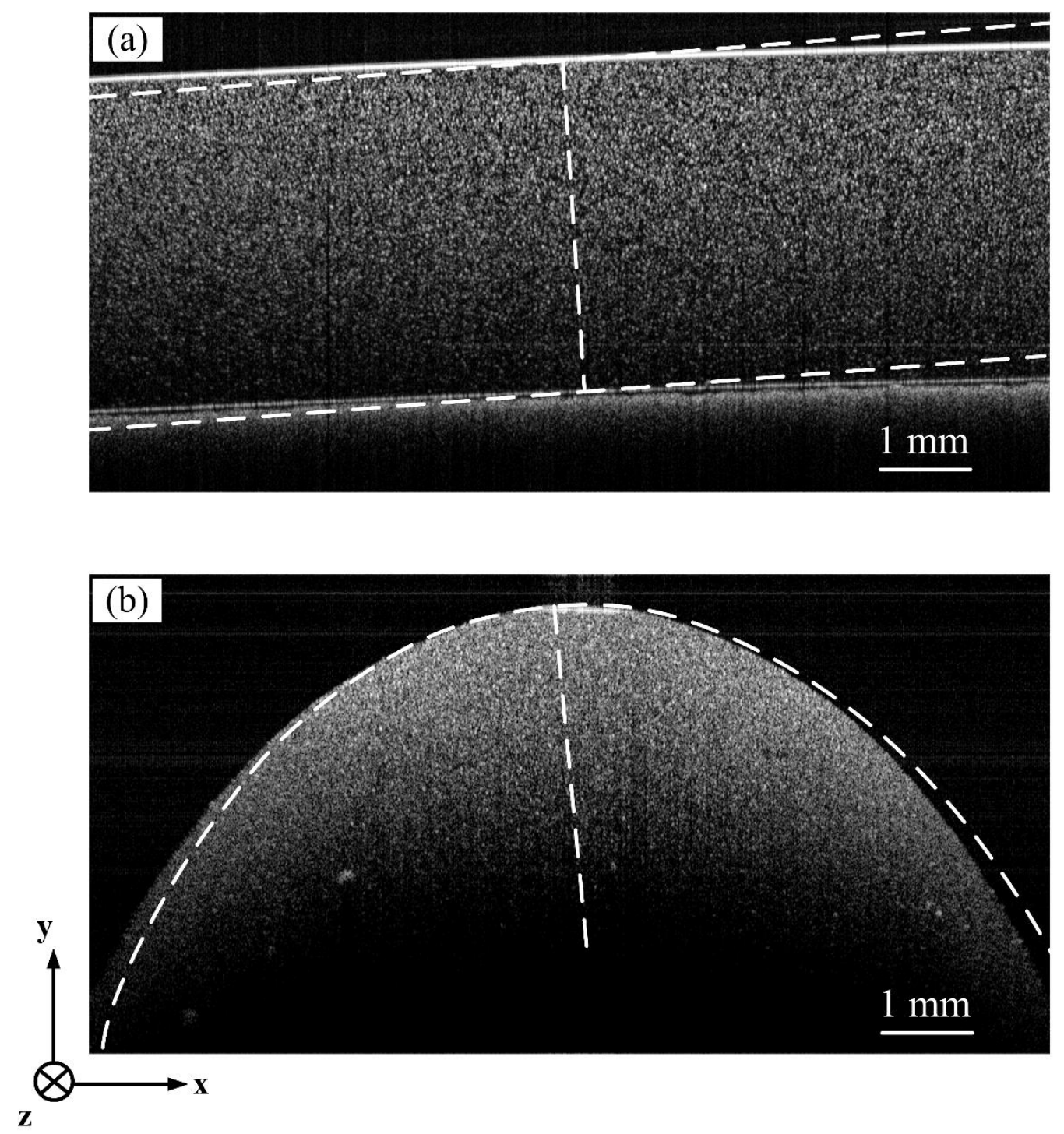

Fig 6. Central z-slices of the OCT reconstructed volumes of: (a) flat and (b) hemispherical silicone rubber phantoms.

Dashed lines indicate the samples interface after rotation of $0.5^{\circ}$.

No smoothing was applied. In Fig. 7, the strain and rotation maps before and after refraction correction are shown for $\varepsilon_{x x}, \varepsilon_{y y}, \varepsilon_{x y}$ and $\omega_{x y}$ for a central $z$-slice of the flat phantom. The mean and standard deviation values of these strain and rotation components are listed in Table 1. It can be observed from Fig. 7(a) and Table 1 that the strain standard deviations of $\varepsilon_{x x}$ and $\varepsilon_{y y}$ before refraction correction are around $5 \times 10^{-4}$. This is close to the strain noise level that has been studied in previous work [45] through stationary and rigid body translation tests, around $4 \times 10^{-4}$ for a sub-volume size of $36^{3}$, obtained with a phantom made from the same silicone rubber MM240-TV 
and copper particles with an average diameter of $1 \mu \mathrm{m}$. The shear strain component $\varepsilon_{x y}$, however, shows positive values that are higher than the noise level, with a mean of $1.3 \times 10^{-3}$. Regarding rotation, $\omega_{x y}$ shows values that are smaller than the reference value $0.5^{\circ}$, with a mean of $0.32^{\circ}$. These results indicate that refraction induced distortion effectively introduces errors to DVC measurements, especially for shear strain and rotational components. After refraction correction, as shown in Fig 7(b) and Table 1, $\varepsilon_{x y}$ drops to the strain noise level and $\omega_{x y}$ approaches the reference $0.5^{\circ}$, with a mean of $0.49^{\circ}$. Similar results were obtained for the hemispherical phantom, with $\varepsilon_{x y}$ reduced from $\sim 2.6 \times 10^{-3}$ to noise level and $\omega_{x y}$ increased from $\sim 0.25^{\circ}$ to $\sim 0.43^{\circ}$ after refraction correction, as can be observed in Fig. 8 and Table 2. These results indicate that DVC measurements are effectively adjusted after refraction correction.

It should be pointed out that there is an increase in standard deviation for the strain and rotation components, as can be seen in Table 1 and Table 2. This is probably due to the interpolation process involved in the refraction correction algorithm.

Table 1. Mean and standard deviation values of the strain and rotation components for the flat phantom rigid body rotation test before and after refraction correction.

\begin{tabular}{|c|c|c|}
\hline & Before correction & After correction \\
\hline$\varepsilon_{x x}$ & Mean: $-2.8 \times 10^{-5}$ & Mean: $5.9 \times 10^{-5}$ \\
& Std: $4.7 \times 10^{-4}$ & Std: $5.8 \times 10^{-4}$ \\
\hline$\varepsilon_{y y}$ & Mean: $1.5 \times 10^{-4}$ & Mean: $2.8 \times 10^{-4}$ \\
& Std: $4.3 \times 10^{-4}$ & Std: $5.5 \times 10^{-4}$ \\
\hline$\varepsilon_{x y}$ & Mean: $1.3 \times 10^{-3}$ & Mean: $2.0 \times 10^{-4}$ \\
& Std: $9.2 \times 10^{-4}$ & Std: $7.4 \times 10^{-4}$ \\
\hline$\omega_{x y}$ & Mean: $-0.32^{\circ}$ & Mean: $-0.49^{\circ}$ \\
& Std: $0.053^{\circ}$ & Std: $0.067^{\circ}$ \\
\hline
\end{tabular}


Table 2. Mean and standard deviation values of the strain and rotation components for the hemispherical phantom rigid body rotation test before and after refraction correction.

\begin{tabular}{|c|c|c|}
\hline & Before correction & After correction \\
\hline$\varepsilon_{x x}$ & Mean: $-2.9 \times 10^{-4}$ & Mean: $-2.0 \times 10^{-4}$ \\
& Std: $1.2 \times 10^{-3}$ & Std: $1.7 \times 10^{-3}$ \\
\hline$\varepsilon_{y y}$ & Mean: $1.9 \times 10^{-4}$ & Mean: $-5.6 \times 10^{-4}$ \\
& Std: $9.0 \times 10^{-4}$ & Std: $1.5 \times 10^{-3}$ \\
\hline$\varepsilon_{x y}$ & Mean: $2.6 \times 10^{-3}$ & Mean: $1.6 \times 10^{-4}$ \\
& Std: $1.2 \times 10^{-3}$ & Std: $2.3 \times 10^{-3}$ \\
\hline$\omega_{x y}$ & Mean: $-0.25^{\circ}$ & Mean: $-0.43^{\circ}$ \\
& Std: $0.069^{\circ}$ & Std: $0.13^{\circ}$ \\
\hline
\end{tabular}
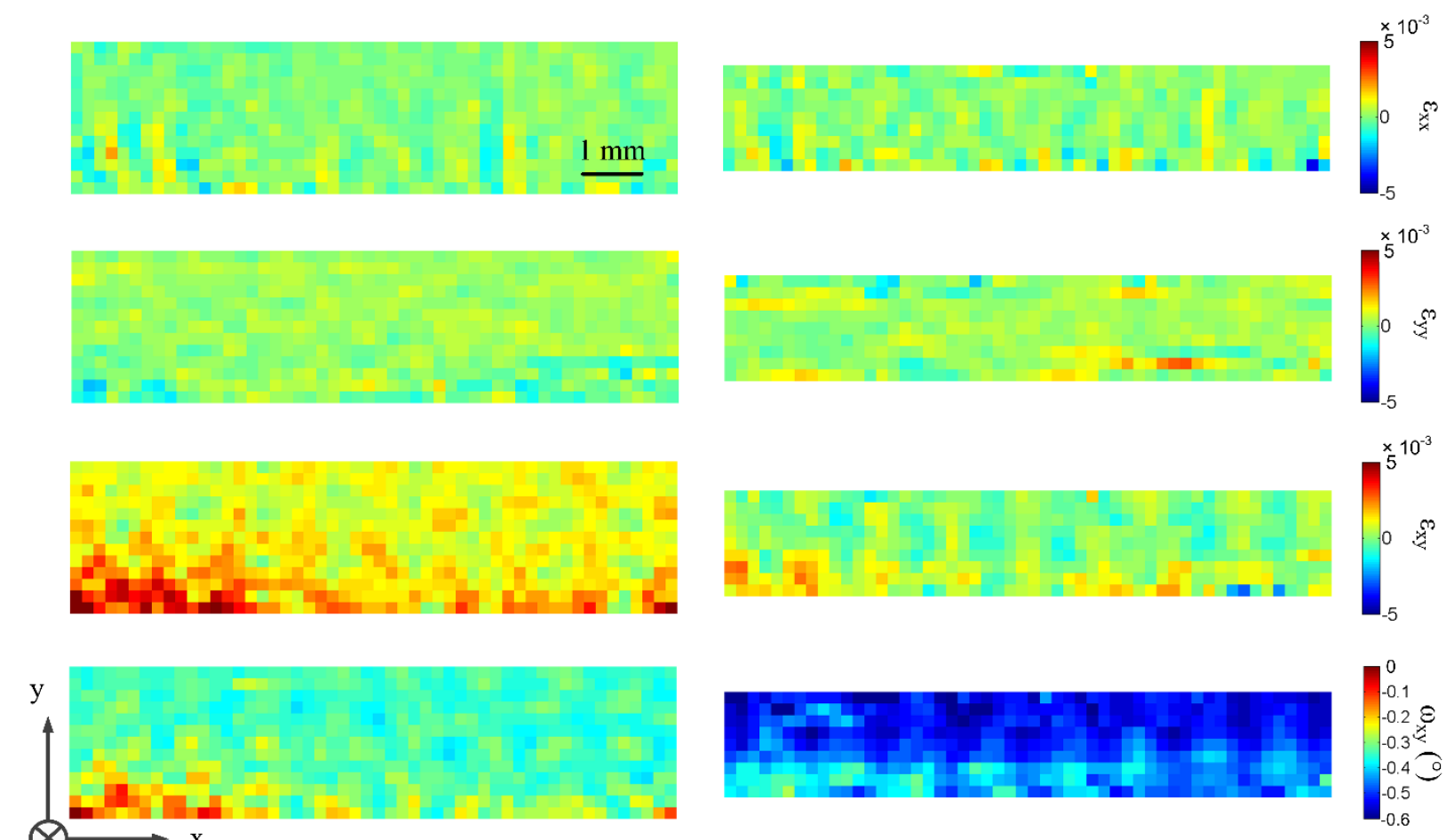

(a)

(b)

Fig 7. Strain and rotation maps for the flat phantom tilt test: (a) before and (b) after refraction correction, $36^{3}$-voxel sub-volume, $50 \%$ overlap. 

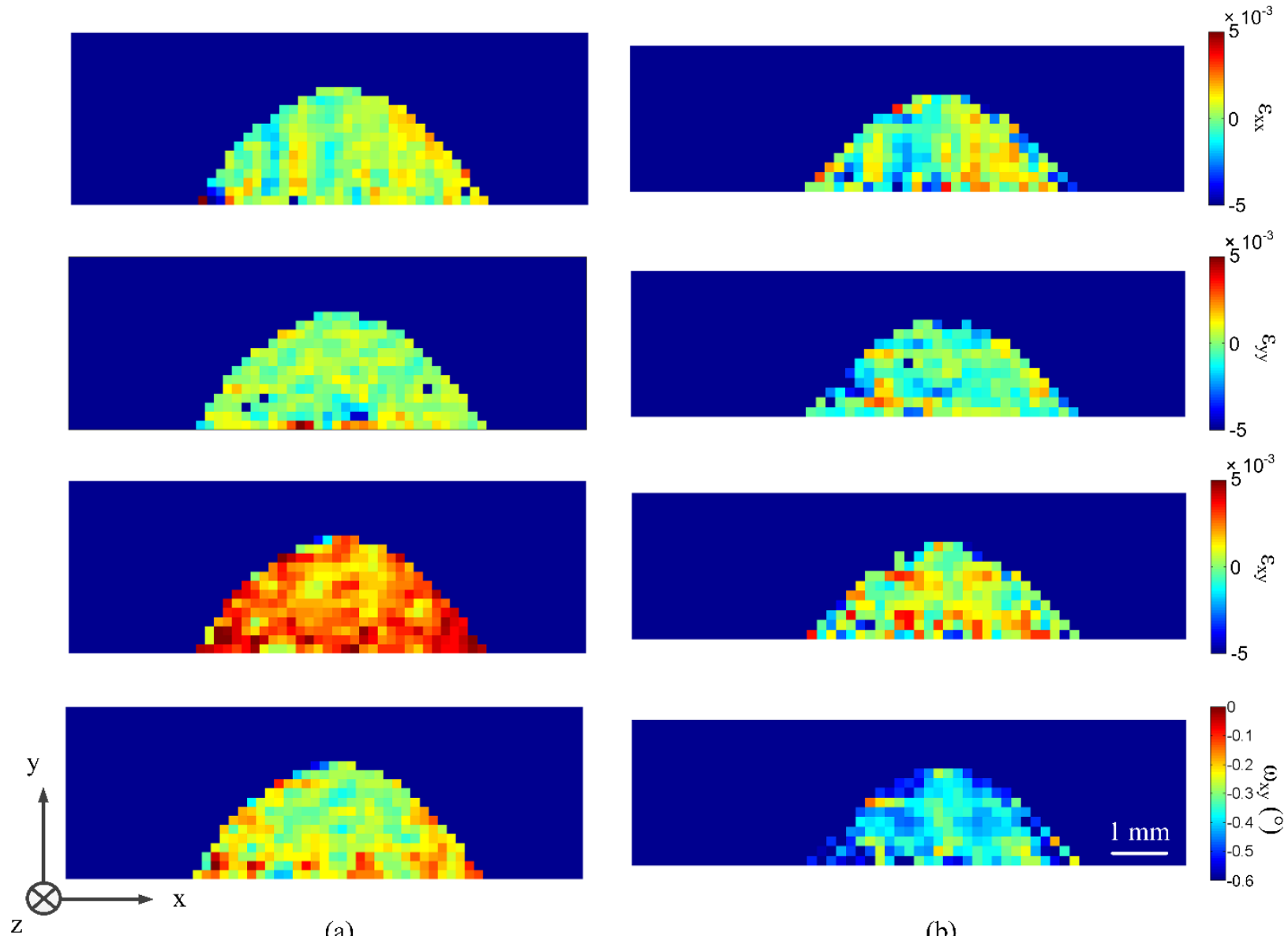

(a)

(b)

Fig 8. Strain and rotation maps for the hemispherical phantom tilt test: (a) before and (b) after refraction correction, 363-voxel sub-volume, 50\% overlap.

\subsection{Finite element modeling of corneal inflation test}

In order to visualize the displacement and strain distributions that we expect through the thickness of the cornea under inflation, a simple finite element model was developed using the commercial software package ABAQUS $6.11^{\circledR}$. The simulation results are aimed at a qualitative comparison with those measured with DVC. According to ref. [13], the porcine cornea behaves linearly while the intraocular pressure remains below $30 \mathrm{mmHg}$. Therefore, a linear elastic material with Young's modulus $E=0.29 \mathrm{MPa}$ and Poisson's ratio $v=0.49$ as in ref. [61] was chosen for the FE model. A study by Anderson, El-Sheikh [13] shows that a model that assumes constant corneal thickness only results in a very small average change in predictions compared to the model with actual thickness variation. Therefore, in the present work, a constant corneal thickness of $1.53 \mathrm{~mm}$ was chosen for the sake of simplicity. A 
first-order reduced-integration element type was selected for the FE model. The corneal volume with FE mesh and a cross section are shown in Fig. 9. The peripheral boundary was fixed while a uniform normal pressure was applied to the inner surface to simulate the intraocular pressure. The model was inflated from $15 \mathrm{mmHg}$ to $18.75 \mathrm{mmHg}$. The displacement and strain simulation results were then compared against the DVC measurements in a common coordinate system.
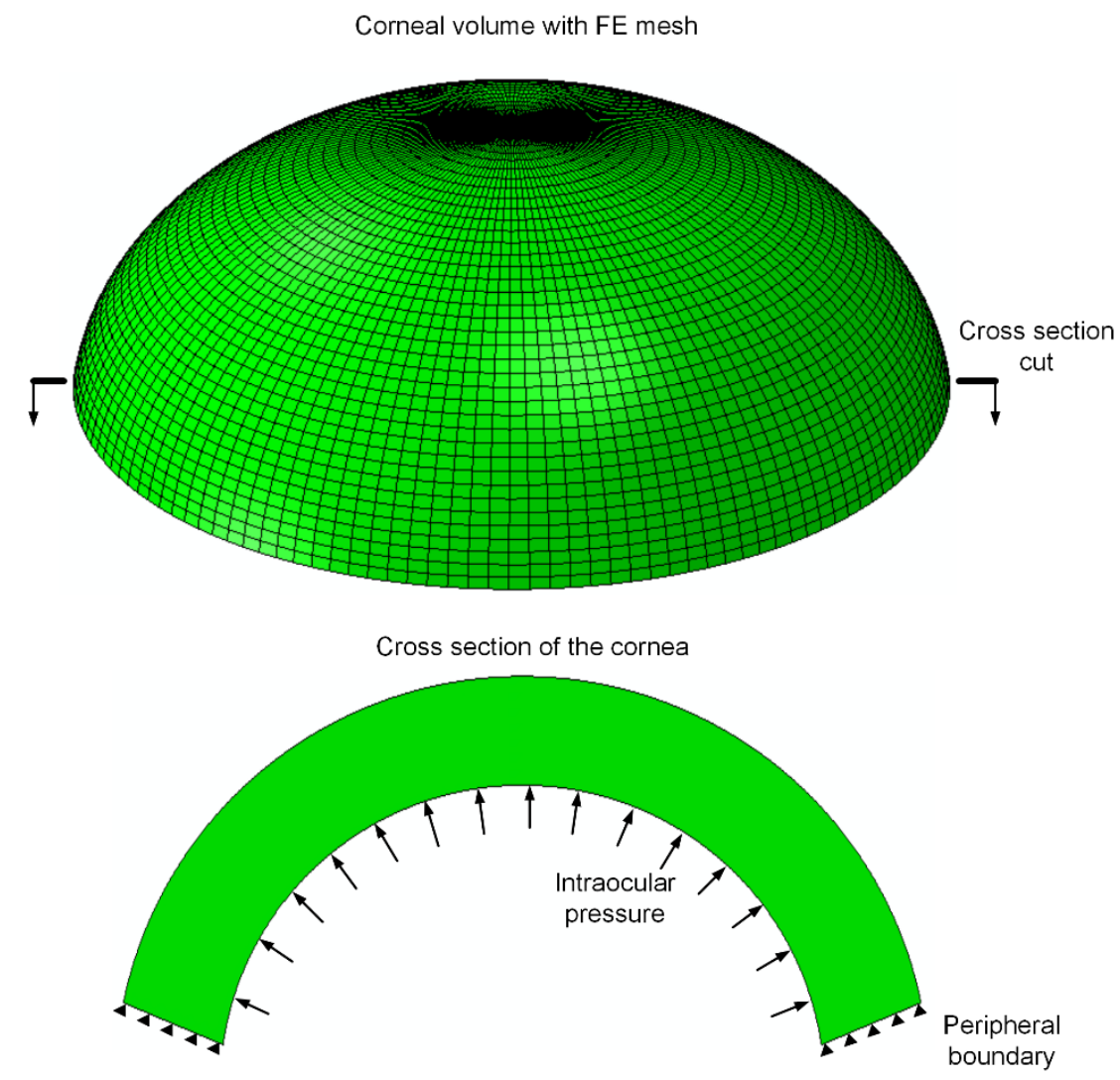

Fig 9. FE model of the porcine cornea inflation test.

\section{Results and discussions}

In the present study, considering the relatively small thickness of the porcine cornea to the spatial resolution of the SS-OCT system, $24^{3}$-voxel sub-volumes with $50 \%$ overlap were used to perform DVC. This sub-volume size can enhance the strain spatial resolution; nevertheless, it increases strain noise level, as proposed in [45], where the noise level increases from around $4 \times 10^{-4}$ to around $6 \times 10^{-4}$ when the sub-volume size reduces from $36^{3}$ to $24^{3}$. These 
noise levels however are about one order of magnitude below the strain levels due to deformation in the inflation test. Fig. 10(a) illustrates the DVC correlation coefficient map for the central $z$-slice of the porcine cornea. In this correlation coefficient map, values in the central region are over 0.8. In the peripheral bottom regions, however, the correlation coefficient values are below 0.6 and thus highly unreliable. This is due to a loss of speckle contrast in those regions (caused by a combination of spectral roll-off, transmission of light at the air/specimen interface and material scattering) as highlighted in Fig. 10(b), where noise becomes dominant and the correlation coefficient is significantly reduced. Therefore, DVC results in the peripheral bottom regions, where the correlation coefficient values are below 0.6, are cropped out later on in this paper to enable a better visualization of the displacement and strain distributions.
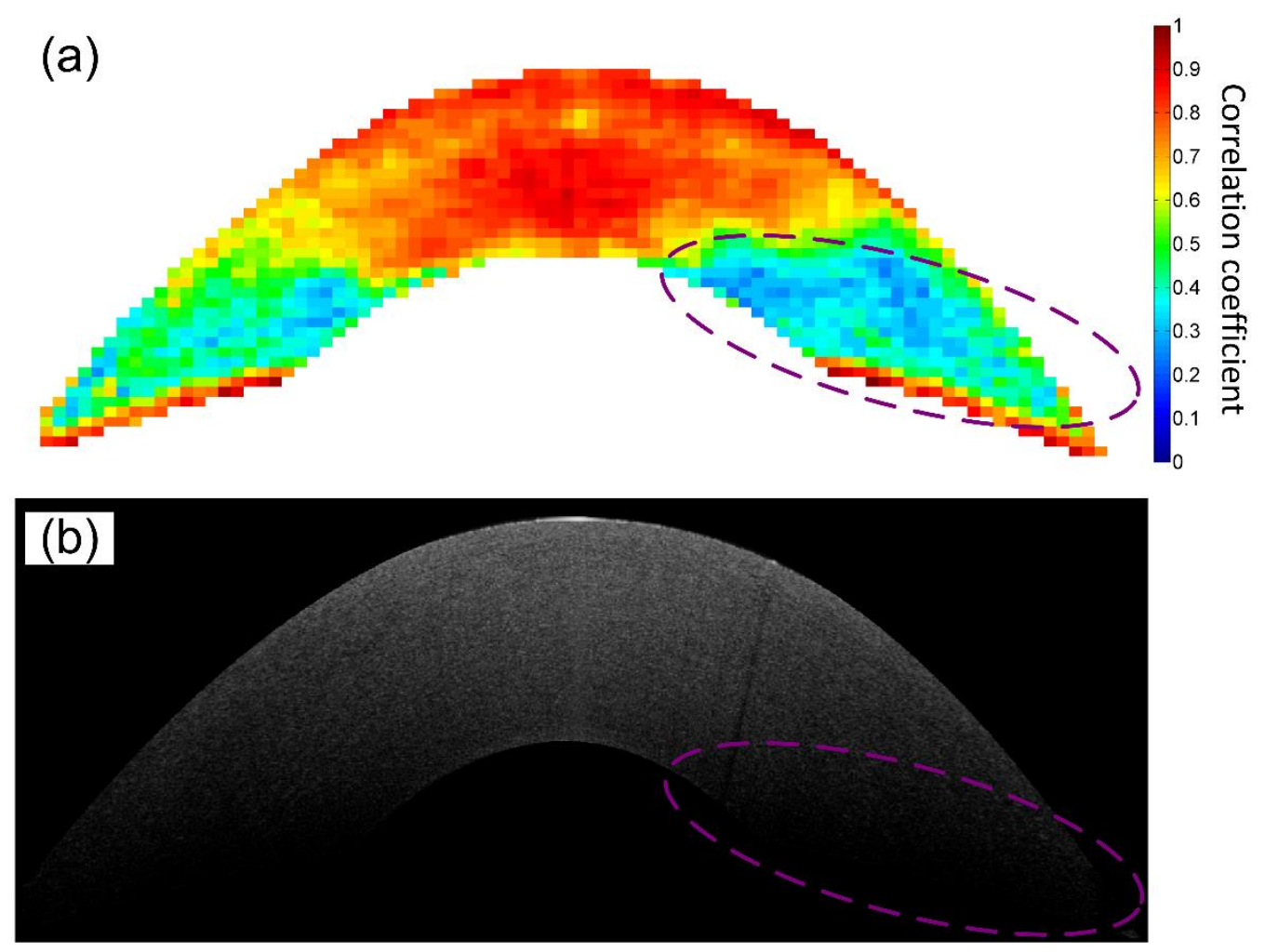

Fig 10. (a) Correlation coefficient map for the central $z$-slice of the porcine cornea inflated from 15 to $18.75 \mathrm{mmHg}$ with $24^{3}$ voxels sub-volume and 50\% overlap, (b) illustration for low speckle contrast in the raw OCT image.

The distributions of different displacement components are presented in 3D view in the first row of Fig. 11. Here, $z$ slices (cut in $x y$-planes) are shown. For the sake of legibility, these displacement maps are only plotted every five $z$ - 
slices. It should be mentioned that only the upper part of the cornea around the apex is shown, where the correlation coefficient is higher. In order to better visualize the distributions, zoomed displacement maps for a transverse $y$-slice (cut in $x z$-plane) are presented in the second row of Fig. 11. The results in columns (a), (b), (c) correspond to $U_{x}, U_{y}$, $U_{z}$, respectively. Displacement maps for the central $z$-slice of the corneal volume are illustrated in Fig. 12(a). In these figures, $U_{y}$ represents the axial (through-thickness) displacement, while $U_{x}$ and $U_{z}$ represent the horizontal displacements along the $x$ - and $z$-directions, respectively. It can be clearly seen that the corneal volume deforms in different directions when the pressure increases. In Fig. 12(b), the corresponding displacement maps for the central $z$-slice obtained with the FE model are shown with the DVC results for comparison. From the DVC results, it can be seen that the horizontal displacement $U_{x}$ is close to zero near the central region, and increases symmetrically away from the apex. The half positive and half negative displacement distribution is expected from the FE model, a result of the hydrostatic pressure loading. The distribution of the horizontal displacement $U_{z}$ is the same as $U_{x}$ due to the rotational symmetry around the $y$-axis, but in this plane ( $z$-slice), it is zero as expected. The maximum $U_{y}$ is located near the endothelium, and $U_{y}$ decreases from the endothelium to the epithelium, which is compatible with a compressive state through the thickness. Qualitatively, the measured displacement maps are consistent with the displacements predicted by the FE model as can be observed in Fig .12. Nevertheless, in Fig .12, there is a difference in the $U_{y}$ amplitudes between the DVC and the FE results, which is probably due to a rigid body movement of the experimental set-up.

As for the displacement, the strain maps for the same 3D view and the transverse $y$-slice are shown in Fig. 13, while the strain maps for the central $z$-slice are shown in Fig .14(a). The measured strains show a good overall agreement in terms of spatial distribution with those predicted by the FE model, as shown in Fig. 14(b). The distribution of the normal strain components $\varepsilon_{x x}$ and $\varepsilon_{z z}$ indicates a tensile deformation along the horizontal $x$ and $z$-axes while that of $\varepsilon_{y y}$ indicates a compressive deformation along the vertical $y$-axis which dominates the central region. The color bars in Figs. 13 and 14 show maximum absolute strains of about 2\%, which exceed the strain standard deviation due to noise and interpolation errors of about $0.2 \%$ as shown in Tables 1 and 2 . 


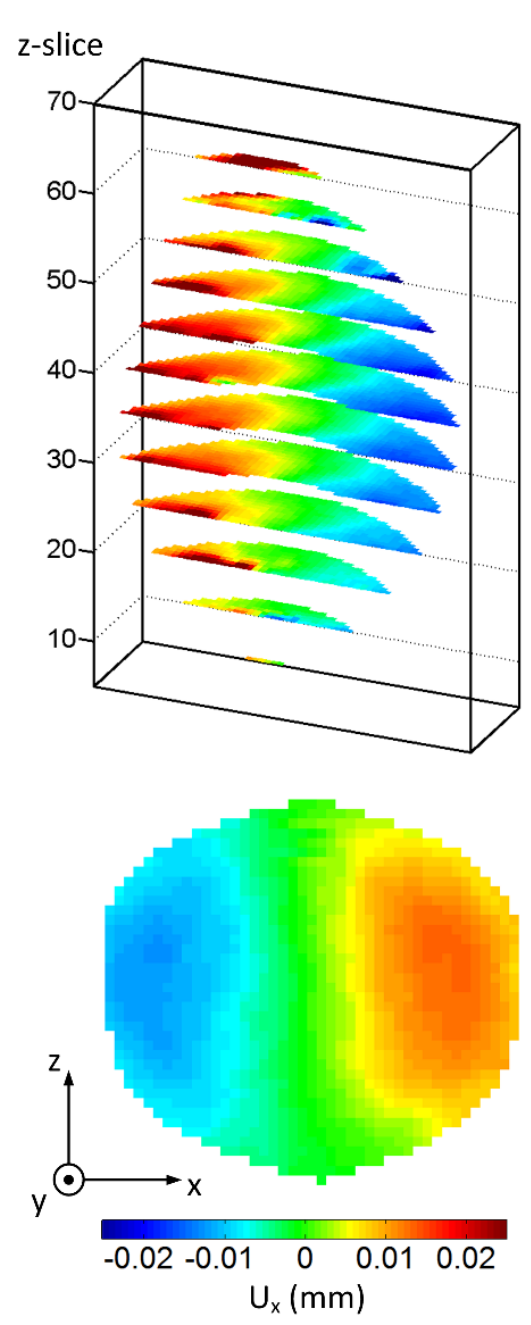

(a)
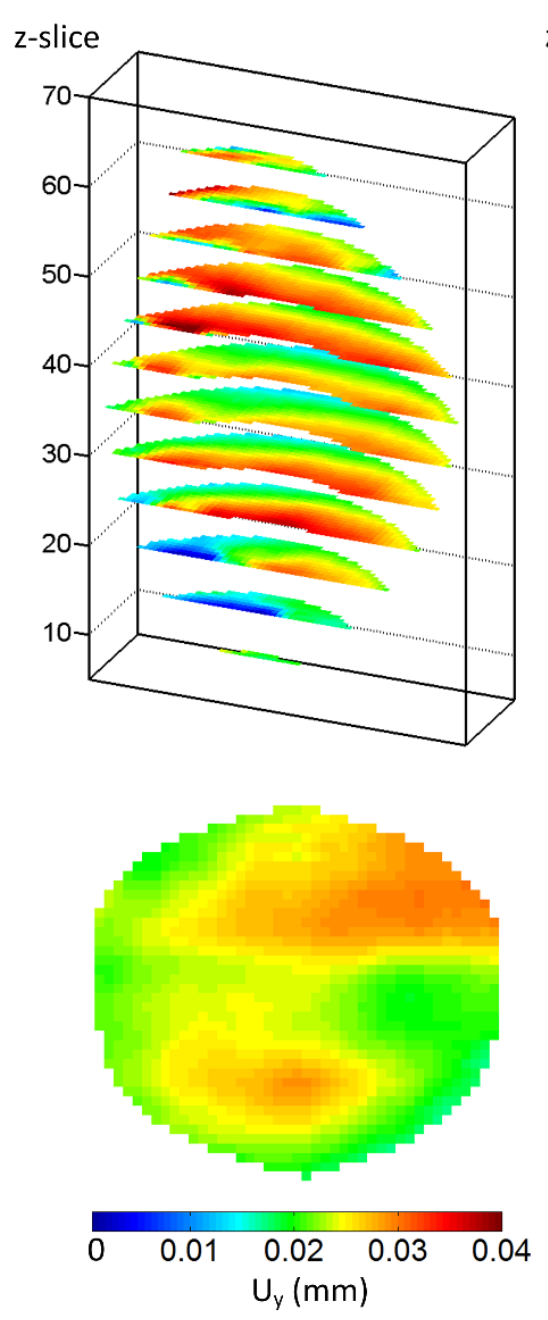

(b)

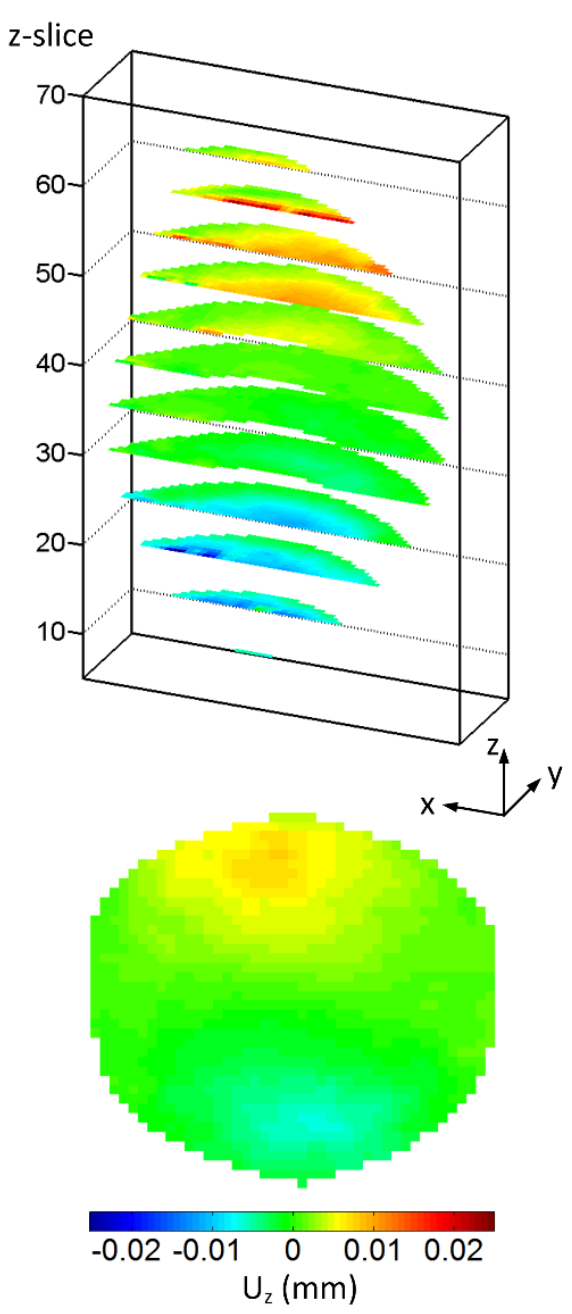

(c)

Fig 11. Displacement maps measured with DVC for the porcine cornea inflated from 15 to $18.75 \mathrm{mmHg}$ with $24^{3}$ voxels sub-volume and 50\% overlap. The first row illustrates the results in $3 \mathrm{D}$ view. The second row illustrates the results in a $y$-slice, $0.76 \mathrm{~mm}$ below the corneal apex. Columns (a), (b), (c) correspond to $U_{x}, U_{y}, U_{z}$, respectively. 


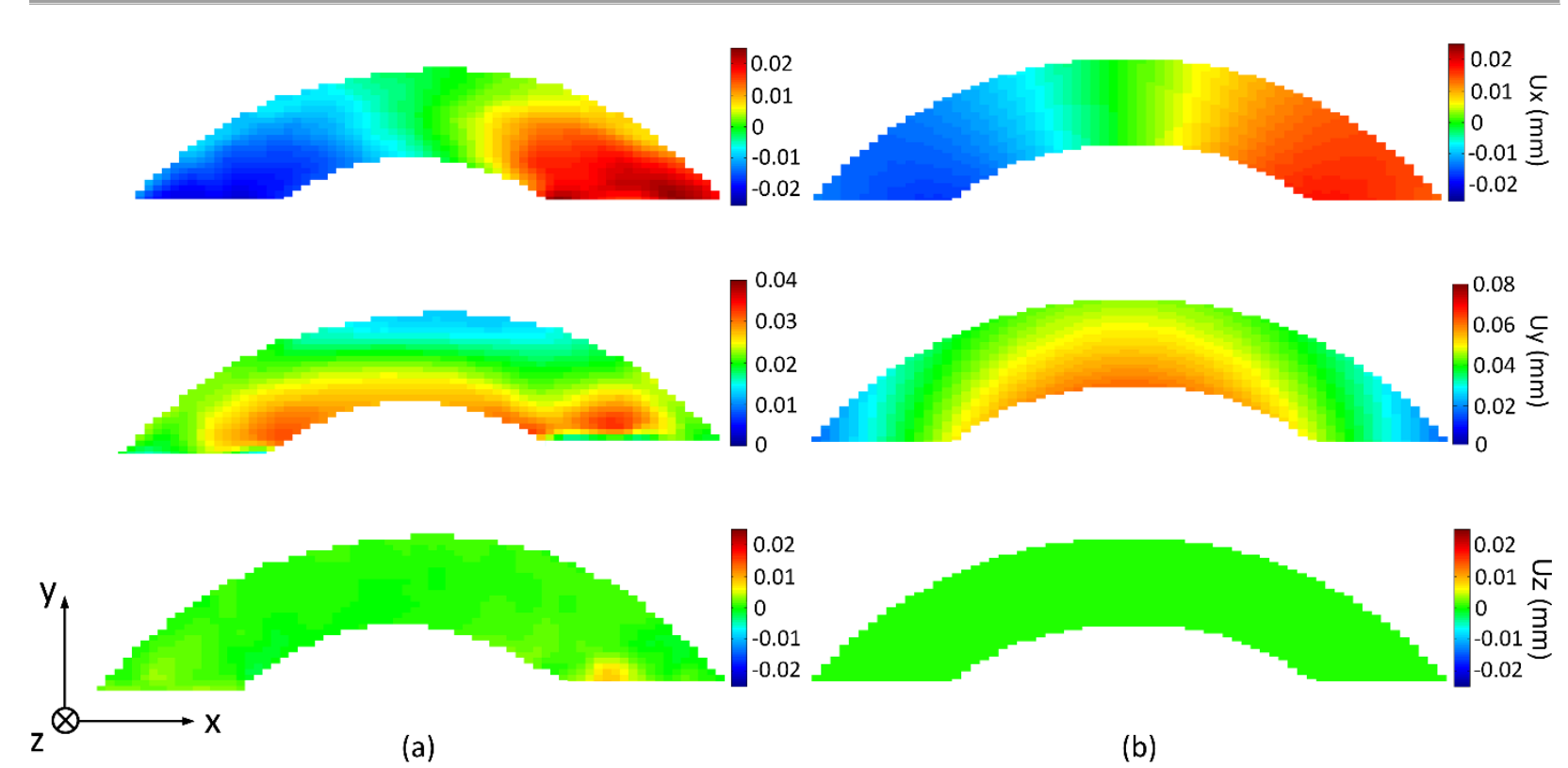

Fig 12. (a) Displacement maps measured with DVC for the central $z$-slice of the porcine cornea inflated from 15 to $18.75 \mathrm{mmHg}$ with $24^{3}$ voxels sub-volume and $50 \%$ overlap, and (b) simulation results.

Although the experimental results show a good overall agreement with the results from the FE model, differences can still be seen between them, especially in the strain maps. Take the strain maps of $\varepsilon_{x x}$ in Fig. 14 as an example. While the strain distribution in the FE model is smooth, with the maximal tension strain in the inner central region and decreasing gradually from centre to periphery, the measured strain shows apparent inhomogeneity and noise with a small background trend that is not clearly observed in the simulated results. These observations are also apparent in the strain components $\varepsilon_{y y}$ and $\varepsilon_{z z}$.

It should be pointed out that the FE model in the present study was developed just for a qualitative comparison with the DVC results to visualize the spatial distribution of the displacement and strain fields. It is not necessarily more accurate than the DVC results since the input material constitutive parameters selected from literature can be very different from the real parameters of the cornea specimen. A thorough FE model of the cornea will require full knowledge of the material constitutive parameters, which could be identified via inverse problem solutions. For the present results, the depth-resolved displacement and strain experimental data measured around the apical region seem appropriate to feed into inverse problem solutions (i.e. FEMU, VFM, CCM) for material characterization purposes. This is beyond the scope of the present work but will be pursued in the future. 


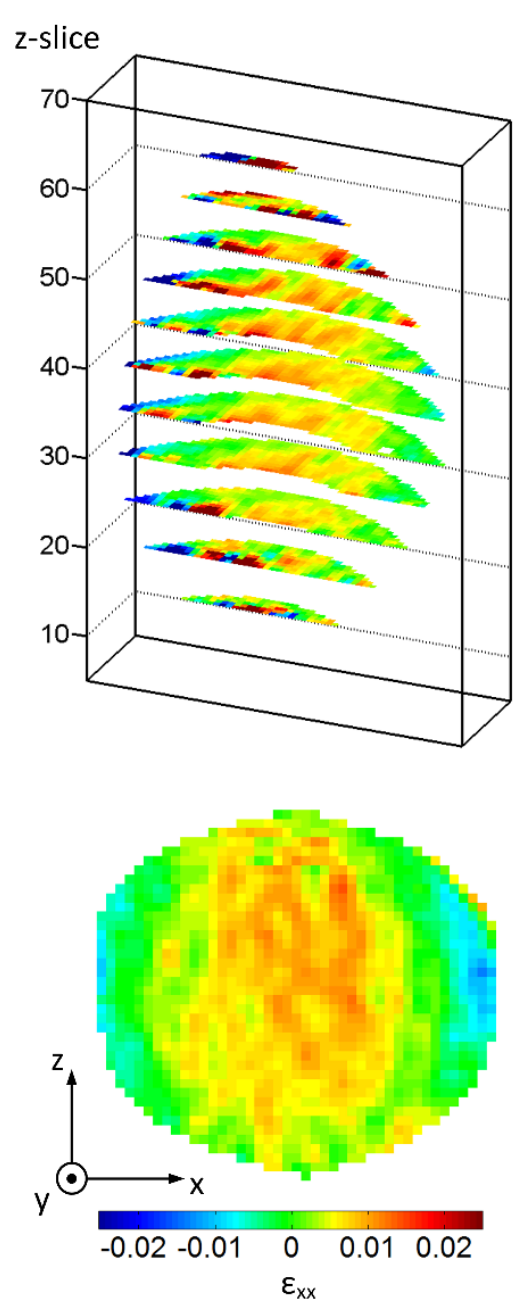

(a)
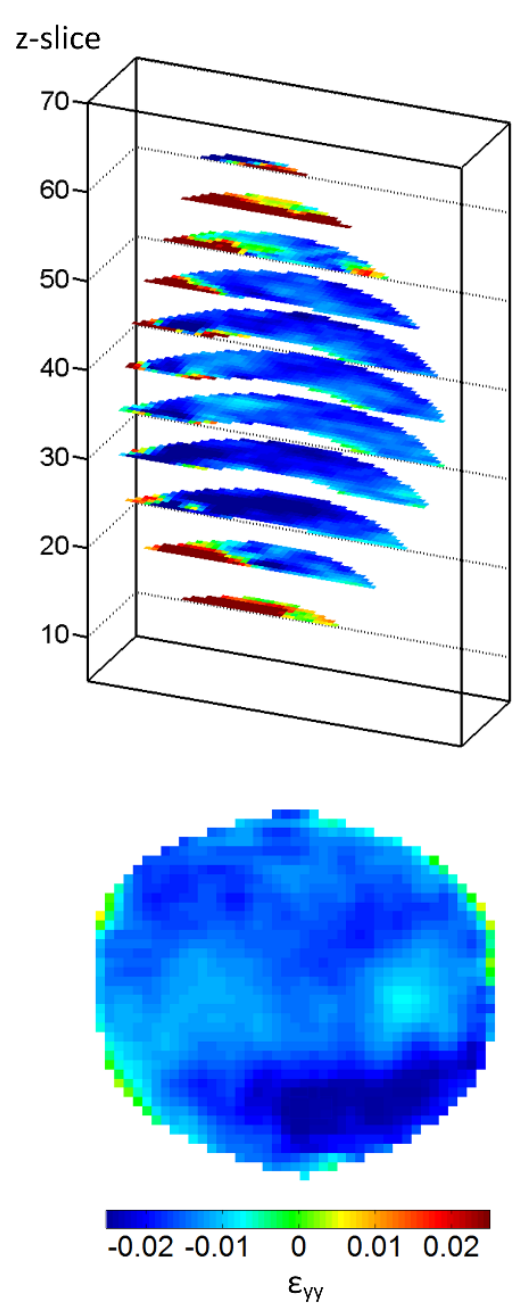

(b) z-slice

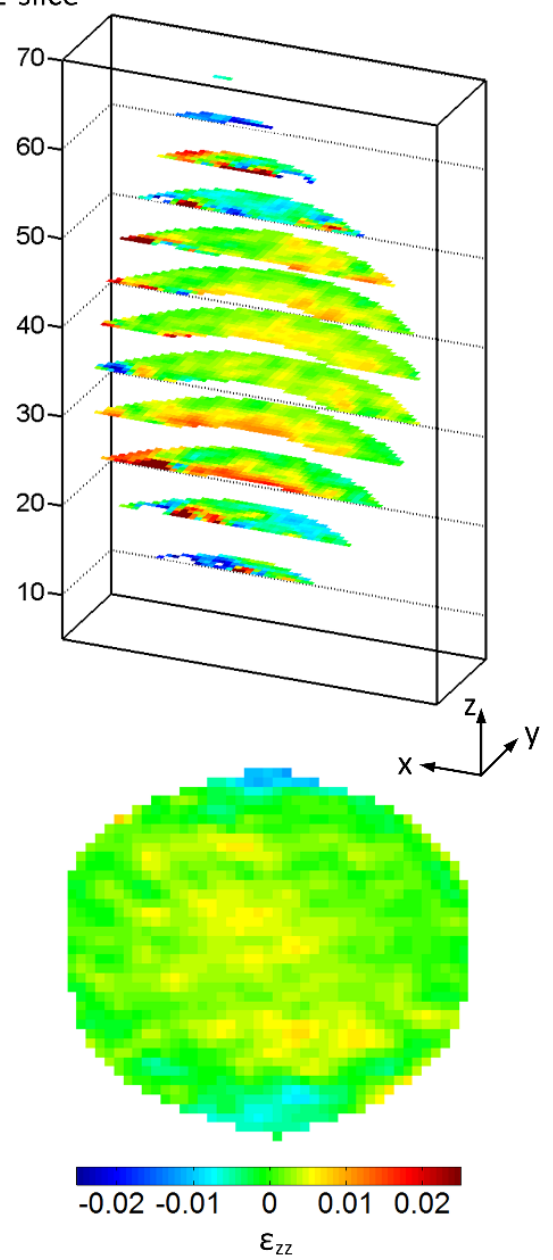

(c)

Fig 13. Strain maps measured with DVC for the porcine cornea inflated from 15 to $18.75 \mathrm{mmHg}$ with $24^{3}$ voxels sub-volume and 50\% overlap. The first row illustrates the results in 3D view. The second row illustrates the results in a $y$-slice, $0.76 \mathrm{~mm}$ below the corneal apex. Columns (a), (b), (c) correspond to $\varepsilon_{x x}, \varepsilon_{y y}, \varepsilon_{z z}$, respectively. 


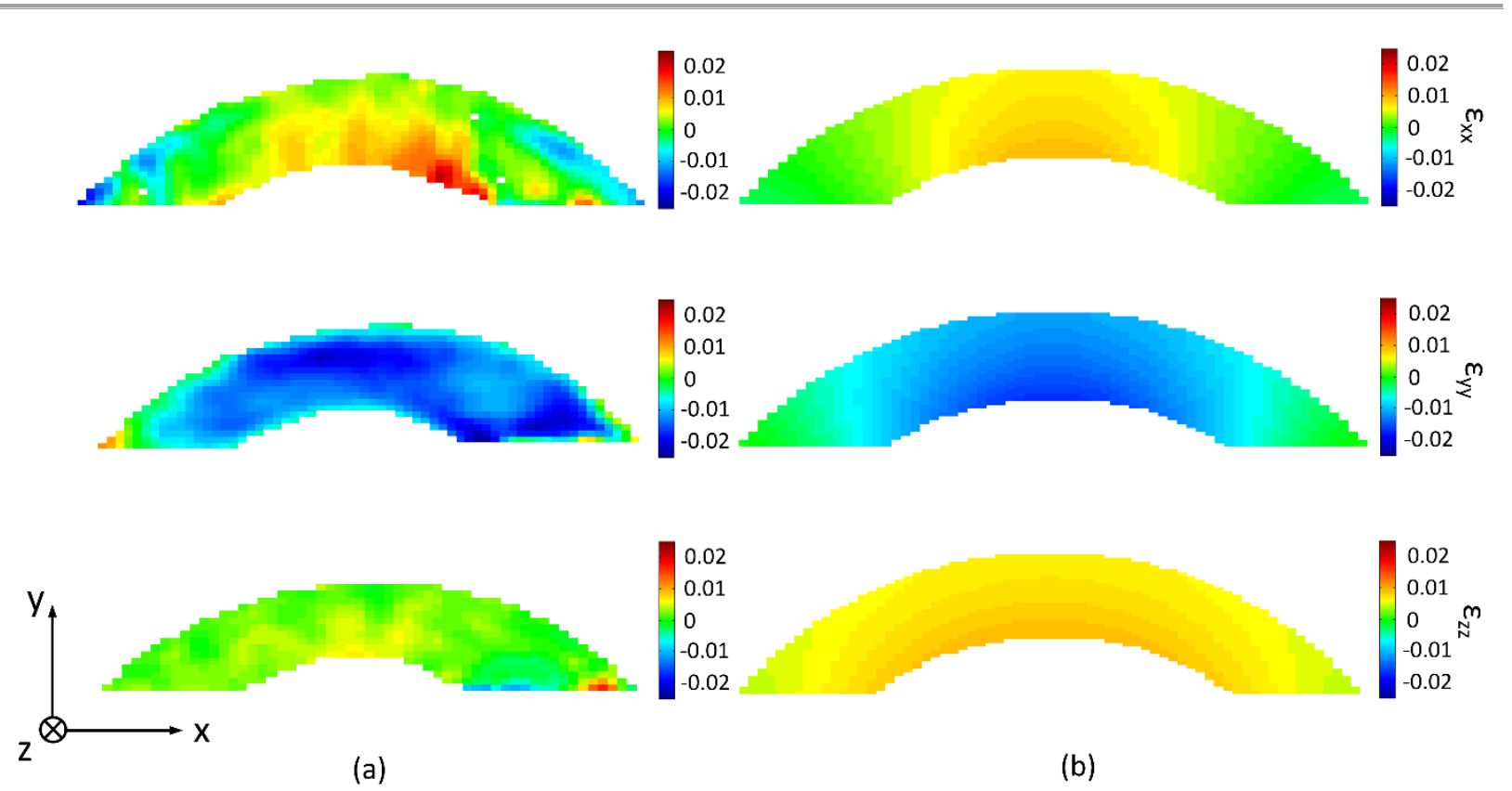

Fig 14. (a) Strain maps measured with DVC for the central $z$-slice of the porcine cornea inflated from 15 to 18.75 mmHg with $24^{3}$ voxels sub-volume and $50 \%$ overlap, and (b) simulation results.

In order to investigate whether the difference between the DVC and the FE distributions is due to material heterogeneity or measurement errors, an artificial corneal phantom with homogeneous material properties was fabricated using silicone rubber MM240-TV A/B (Young's modulus E=1.88 MPa and Poisson's ratio v=0.49 from the material technical data sheet). A rubber base and a hardener were mixed to a ratio of 10:1. Titanium oxide particles were seeded into the silicone rubber mixture to supply a speckle contrast. The mixture was then put into a special mould previously designed to replicate the average dimensions of the Arizona eye model [62]. Its outer edge spherical diameter and central thickness are $15.6 \mathrm{~mm}$ and $0.58 \mathrm{~mm}$, respectively. The corneal phantom was inflated from $15 \mathrm{mmHg}$ to $18.75 \mathrm{mmHg}$. Fig. 15 shows the central $z$-slice of the OCT reconstruction in the reference state. DVC was performed using $24^{3}$-voxel sub-volumes with $50 \%$ overlap. As for the porcine cornea, a FE simulation was carried out for the corneal phantom to compare with the DVC results. 


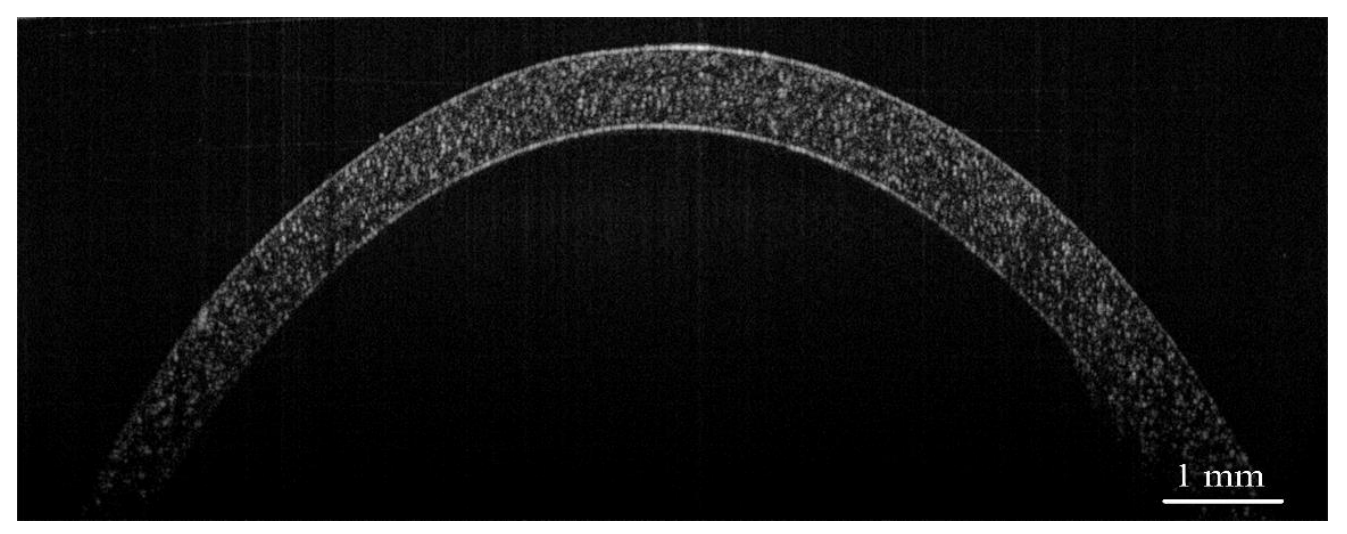

Fig 15. Central $z$-slice of the OCT reconstructed volume of the silicone rubber corneal phantom.

The displacement maps for the central $z$-slice of the corneal phantom and the FE simulation are illustrated in Fig. 16. In Fig 17, the displacement maps are shown for a $y$-slice cut in $x z$-plane, $0.29 \mathrm{~mm}$ below the phantom apex (crosssection A-A as designated in Fig 16). From the comparison between the DVC and the FE results, good agreement can be observed for all the displacement components although it appears that the displacement distributions for the DVC results are slightly off-centred from the phantom apex. Interestingly, when comparing the displacement maps of the phantom in Fig. 17 to those of the porcine cornea in Fig. 11, one can observe that $U_{x}$ and $U_{z}$ show similar amplitudes for the phantom, which is consistent with the FE simulation, whereas $U_{x}$ shows substantially higher amplitude than $U_{z}$ for the porcine cornea. Regarding $U_{y}$, the maximum displacements for the porcine cornea are offcentred and located in peripheral regions. One possible explanation for these is the material anisotropy and heterogeneity of the porcine cornea due to its multi-layer structure and the embedded collagen fibrils arranged in different orientations [63]. 

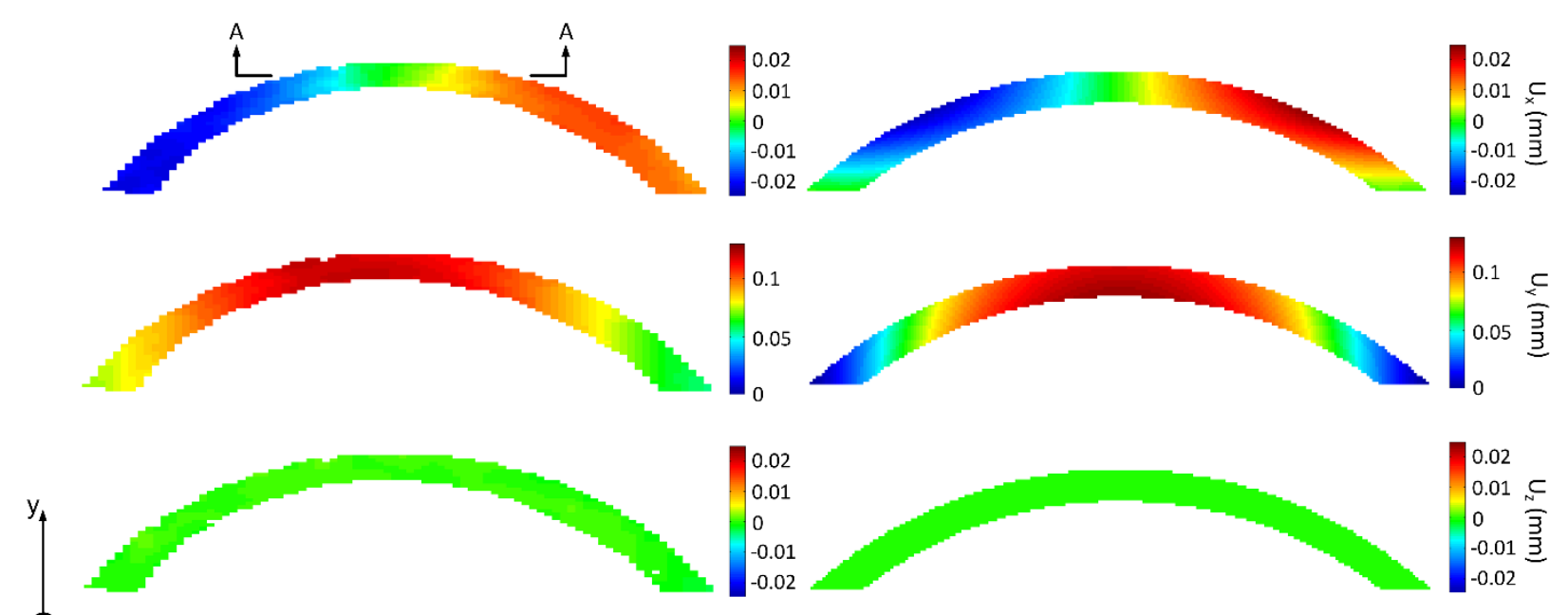

(a)

(b)

Fig 16. (a) Displacement maps measured with DVC for the central $z$-slice of the corneal phantom inflated from 15 to $18.75 \mathrm{mmHg}$ with $24^{3}$ voxels sub-volume and $50 \%$ overlap, and (b) simulation results.

\section{Section A-A}
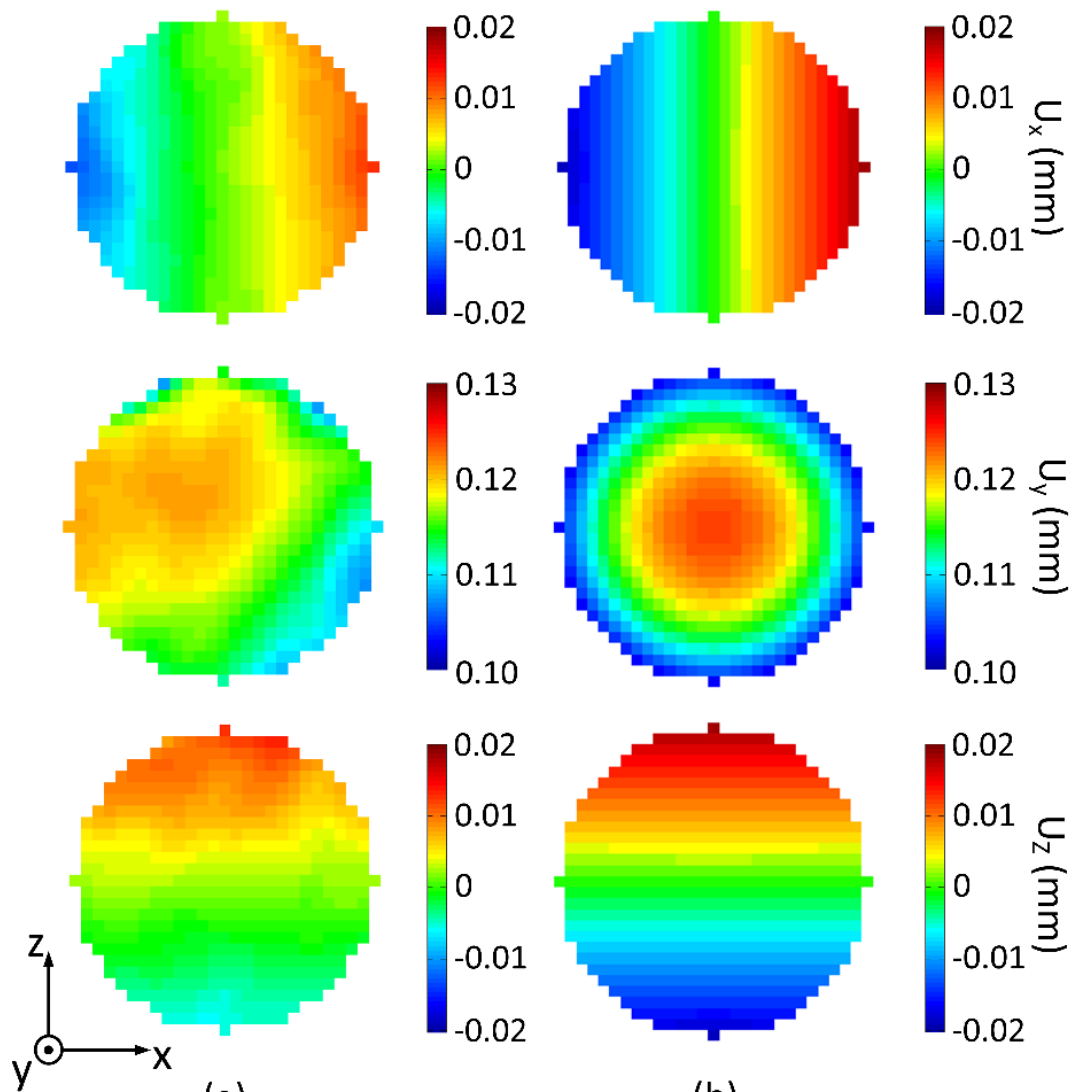

(a)

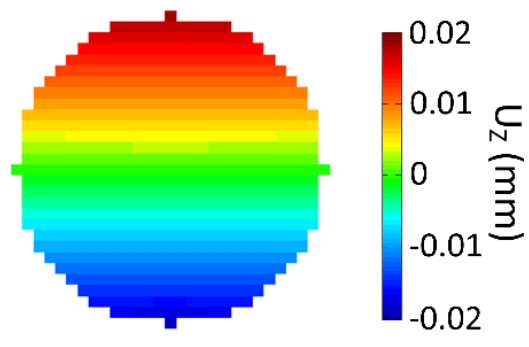

(b)

Fig 17. (a) Displacement maps measured with DVC for the central $y$-slice of the corneal phantom inflated from 15 to $18.75 \mathrm{mmHg}$ with $24^{3}$ voxels sub-volume and $50 \%$ overlap, and (b) simulation results. 
The strain maps for the same $z$-slice and $y$-slice of the corneal phantom and the FE simulation are illustrated in Figs. 18 and 19. Although the general strain distributions of the DVC results are consistent with the simulation results, the DVC results are apparently heterogeneous even though the phantom material is homogeneous. One can clearly see the fringes in the DVC strain maps, which are not expected to present for a homogeneous material. These fringes are due to interpolation bias of the DVC algorithm, and this has been studied in [45]. Briefly, some of the fine speckle patterns in the current OCT reconstructions are spatially undersampled, which, consequently, leads to interpolation bias in displacement. The bias is then amplified when calculating strain, proportional to the slope of the displacement bias, and typically shows up as a Moiré-like fringe pattern. Therefore, the strain uncertainties arising from the stationary and the rigid body motions underestimate the noise floor because of the interpolation bias. It is also interesting to note that in Figs. 18 and 19, the strain values of $\varepsilon_{x x}$ are very close to those of $\varepsilon_{z z}$ for the corneal phantom, which is consistent with the similar displacement amplitudes found for $U_{x}$ and $U_{z}$ in Fig. 17. However, for the porcine cornea, as can be observed in Figs. 13 and 14, the strain values of $\varepsilon_{x x}$ are apparently larger than those of $\varepsilon_{z z}$, corresponding to the larger displacement observed for $U_{x}$ then for $U_{z}$ in Fig. 11. The reason for this has been suggested in the previous paragraph (material anisotropy and heterogeneity). The above results indicate that the heterogeneous displacement and strain distributions observed in the DVC results of the porcine cornea are caused by a combination of material anisotropy and heterogeneity as well as interpolation bias. However, at this stage, the quality of the measurements is not sufficient enough to discriminate them. Future work is needed to improve the measurement quality so that these issues can be studied more clearly. 


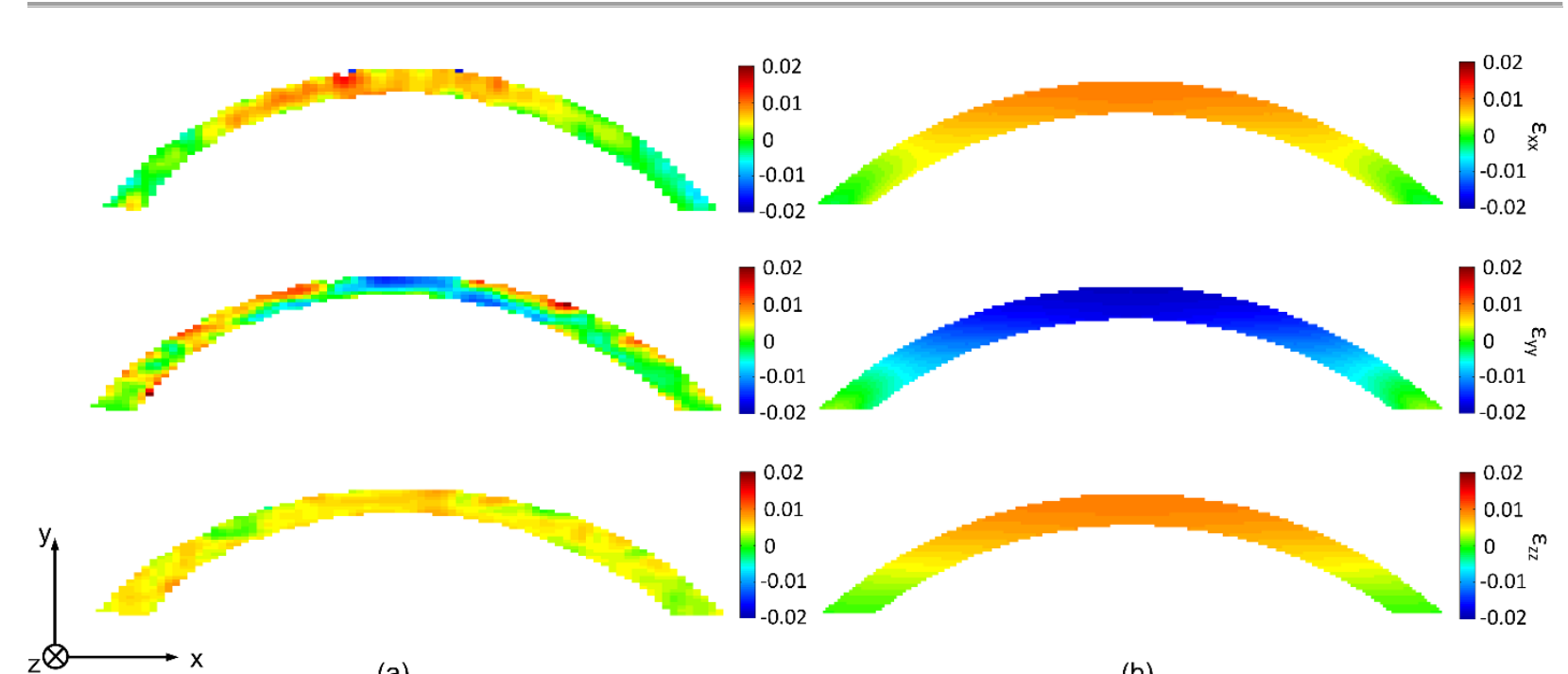

(a)

(b)

Fig 18. (a) Strain maps measured with DVC for the central $z$-slice of the corneal phantom inflated from 15 to 18.75 mmHg with $24^{3}$ voxels sub-volume and 50\% overlap, and (b) simulation results.

\section{Section A-A}

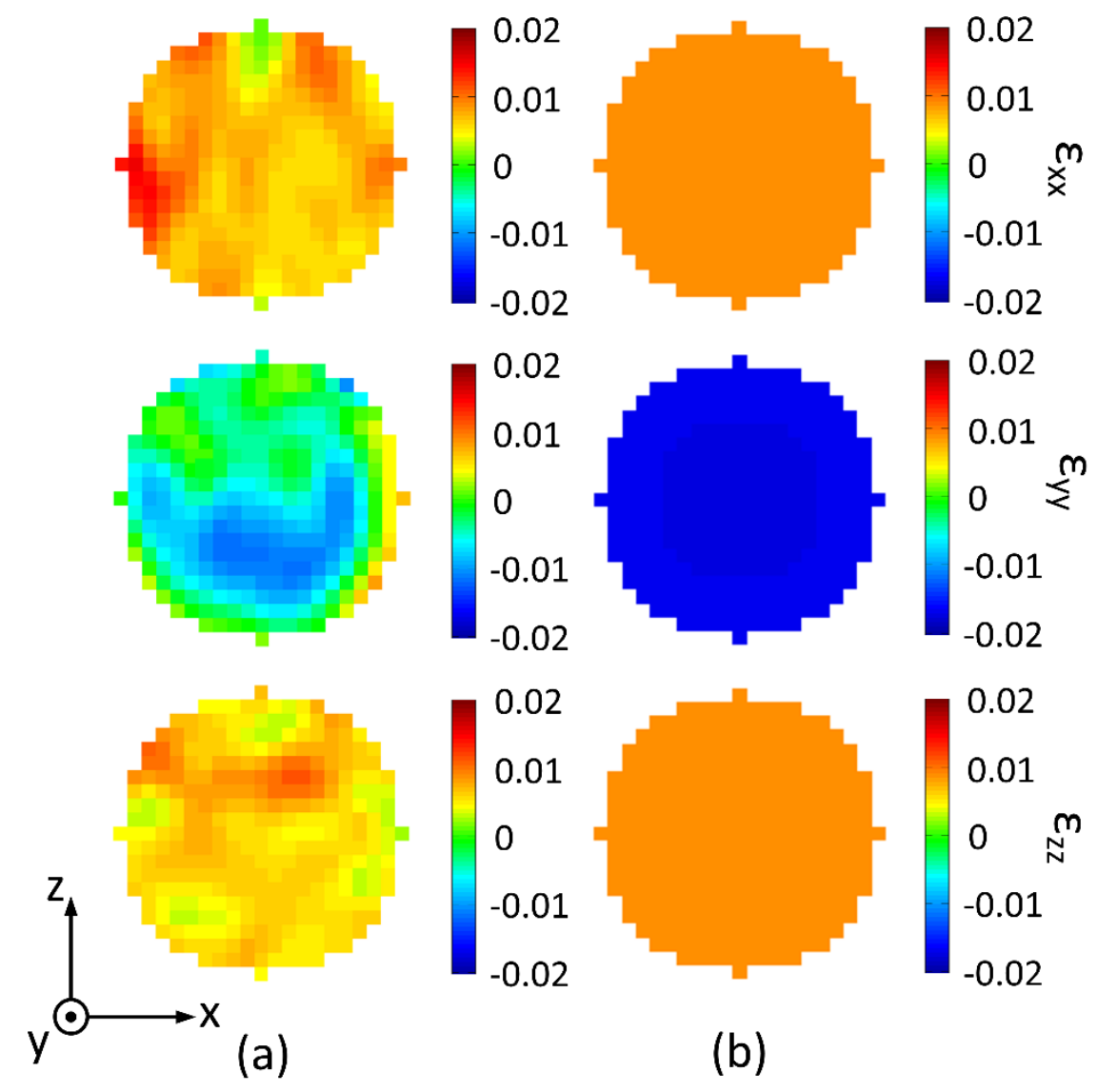

Fig 19. (a) Strain maps measured with DVC for the central $y$-slice of the corneal phantom inflated from 15 to 18.75 mmHg with $24^{3}$ voxels sub-volume and 50\% overlap, and (b) simulation results. 


\section{Conclusions}

In the present study, depth-resolved 3D displacement and strain fields were measured inside a porcine corneal trephinate due to changes in intraocular pressure. First, a method based on Fermat's principle was developed to correct the commonly seen refraction induced distortions in the OCT reconstructions. Based on the rigid body rotation tests of the silicone rubber phantoms, this correction method has proved to be able to adjust DVC measurements. Then, DVC was performed on the refraction corrected OCT corneal data volumes. The measured displacement and strain show a good overall agreement with the results of the simple FE model. The measured fields will be used to identify the elastic constitutive parameters of the cornea through the solution of an inverse problem using FEMU, VFM or CCM. The quality of the data, however, does not seem high enough to investigate the through-thickness dependence of stiffness as more work is required to increase the spatial resolution through the thickness.

\section{Acknowledgments}

The authors would like to thank the China Scholarship Council and the Wolfson School of Mechanical and Manufacturing Engineering, Loughborough University, for their financial support. Professor Pierron gratefully acknowledges support from the Royal Society and the Wolfson Foundation through a Royal Society Wolfson Research Merit Award.

\section{References}

1. Atchison, D.A. and G. Smith, Chapter 2 - Refracting components: cornea and lens, in Optics of the Human Eye, D.A.A. Smith, Editor. 2000, Butterworth-Heinemann: Edinburgh. p. 11-20.

2. Fatt, I. and B.A. Weissman, 6 - Cornea I: Form, Swelling Pressure, Transport Processes, and Optics*, in Physiology of the Eye (Second Edition), I.F.A. Weissman, Editor. 1992, ButterworthHeinemann. p. 97-149.

3. Browning, A.C., et al., The effect of corneal thickness on intraocular pressure measurement in patients with corneal pathology. British Journal of Ophthalmology, 2004. 88(11): p. 1395-1399. 
4. Krachmer, J.H., R.S. Feder, and M.W. Belin, Keratoconus and related noninflammatory corneal thinning disorders. Surv Ophthalmol, 1984. 28(4): p. 293-322.

5. Notara, M., et al., In sickness and in health: Corneal epithelial stem cell biology, pathology and therapy. Exp Eye Res, 2010. 90(2): p. 188-95.

6. $\quad$ Robin, J.B., et al., Peripheral corneal disorders. Surv Ophthalmol, 1986. 31(1): p. 1-36.

7. Tarkkanen, A. and L. Merenmies, Corneal pathology and outcome of keratoplasty in autoimmune polyendocrinopathy-candidiasis-ectodermal dystrophy (APECED). Acta Ophthalmol Scand, 2001. 79(2): p. $204-7$.

8. De Paiva, C.S., et al., The incidence and risk factors for developing dry eye after myopic LASIK. Am J Ophthalmol, 2006. 141(3): p. 438-45.

9. Asejczyk-Widlicka, M., et al., Material properties of the cornea and sclera: a modelling approach to test experimental analysis. J Biomech, 2011. 44(3): p. 543-6.

10. Bryant, M.R. and P.J. McDonnell, Constitutive laws for biomechanical modeling of refractive surgery. J Biomech Eng, 1996. 118(4): p. 473-81.

11. Kwon, T.H., et al., Effect of cornea material stiffness on measured intraocular pressure. $\mathrm{J}$ Biomech, 2008. 41(8): p. 1707-13.

12. Norman, R.E., et al., Finite element modeling of the human sclera: influence on optic nerve head biomechanics and connections with glaucoma. Exp Eye Res, 2011. 93(1): p. 4-12.

13. Anderson, K., A. El-Sheikh, and T. Newson, Application of structural analysis to the mechanical behaviour of the cornea. Journal of the Royal Society Interface, 2004. 1(1): p. 3-15. 
14. Boyce, B.L., et al., Full-field deformation of bovine cornea under constrained inflation conditions. Biomaterials, 2008. 29(28): p. 3896-904.

15. Chu, T.C., W.F. Ranson, and M.A. Sutton, Applications of digital-image-correlation techniques to experimental mechanics. Experimental Mechanics, 1985. 25(3): p. 232-244.

16. Elsheikh, A., D. Alhasso, and P. Rama, Biomechanical properties of human and porcine corneas. Experimental Eye Research, 2008. 86(5): p. 783-790.

17. Ehlers, N., T. Bramsen, and S. Sperling, APPLANATION TONOMETRY AND CENTRAL CORNEAL THICKNESS. Acta Ophthalmologica, 1975. 53(1): p. 34-43.

18. Siganos, D.S., G.I. Papastergiou, and C. Moedas, Assessment of the Pascal dynamic contour tonometer in monitoring intraocular pressure in unoperated eyes and eyes after LASIK. Journal of Cataract \& Refractive Surgery, 2004. 30(4): p. 746-751.

19. Whitacre, M.M., R.A. Stein, and K. Hassanein, The Effect of Corneal Thickness on Applanation Tonometry. American Journal of Ophthalmology, 1993. 115(5): p. 592-596.

20. Luce, D.A., Determining in vivo biomechanical properties of the cornea with an ocular response analyzer. Journal of Cataract \& Refractive Surgery, 2005. 31(1): p. 156-162.

21. Shah, S., et al., Assessment of the biomechanical properties of the cornea with the ocular response analyzer in normal and keratoconic eyes. Investigative Ophthalmology and Visual Science, 2007. 48(7): p. 3026-3031.

22. Hong, J., et al., A new tonometer--the Corvis ST tonometer: clinical comparison with noncontact and Goldmann applanation tonometers. Invest Ophthalmol Vis Sci, 2013. 54(1): p. 659-65. 
23. Ji, C., et al., Dynamic curvature topography for evaluating the anterior corneal surface change with Corvis ST. BioMedical Engineering Online, 2015. 14(1).

24. Koprowski, R., et al., Selected parameters of the corneal deformation in the Corvis tonometer. BioMedical Engineering Online, 2014. 13(1).

25. Fercher, A.F., Optical coherence tomography-development, principles, applications. Zeitschrift für Medizinische Physik, 2010. 20(4): p. 251-276.

26. Gambichler, T., V. Jaedicke, and S. Terras, Optical coherence tomography in dermatology: technical and clinical aspects. Arch Dermatol Res, 2011. 303(7): p. 457-73.

27. Kaluzny, B.J., et al., Spectral optical coherence tomography: a novel technique for cornea imaging. Cornea, 2006. 25(8): p. 960-5.

28. Kiernan, D.F., W.F. Mieler, and S.M. Hariprasad, Spectral-domain optical coherence tomography: a comparison of modern high-resolution retinal imaging systems. Am J Ophthalmol, 2010. 149(1): p. 18-31.

29. Kennedy, B.F., et al., Strain estimation in phase-sensitive optical coherence elastography. Biomedical Optics Express, 2012. 3(8): p. 1865-1879.

30. Kirkpatrick, S.J., R.K. Wang, and D.D. Duncan, OCT-based elastography for large and small deformations. Optics Express, 2006. 14(24): p. 11585-11597.

31. Sun, C., B. Standish, and V.X.D. Yang, Optical coherence elastography: current status and future applications. Journal of Biomedical Optics, 2011. 16(4): p. 043001-043001-12.

32. Wang, R.K., S. Kirkpatrick, and M. Hinds, Phase-sensitive optical coherence elastography for mapping tissue microstrains in real time. Applied Physics Letters, 2007. 90(16): p. 164105. 
33. Zaitsev, V.Y., et al., Elastographic mapping in optical coherence tomography using an unconventional approach based on correlation stability. J Biomed Opt, 2014. 19(2): p. 21107.

34. Ford, M.R., et al., Method for optical coherence elastography of the cornea. Journal of Biomedical Optics, 2011. 16(1): p. 016005-016005-7.

35. Kennedy, B.F., et al., In vivo three-dimensional optical coherence elastography. Optics Express, 2011. 19(7): p. 6623-6634.

36. Li, J., et al. Air-puff OCE for assessment of mouse cornea in vivo. 2014.

37. Twa, M.D., et al., Spatial characterization of corneal biomechanical properties with optical coherence elastography after UV cross-linking. Biomedical Optics Express, 2014. 5(5): p. 1419-1427.

38. Wang, S. and K.V. Larin, Noncontact depth-resolved micro-scale optical coherence elastography of the cornea. Biomedical Optics Express, 2014. 5(11): p. 3807-3821.

39. Manapuram, R.K., et al., In vivo estimation of elastic wave parameters using phase-stabilized swept source optical coherence elastography. J Biomed Opt, 2012. 17(10): p. 100501.

40. Nguyen, T.M., et al. Shear wave elastography of ex vivo human corneas using phase-sensitive optical coherence tomography. in IEEE International Ultrasonics Symposium, IUS. 2014.

41. Nguyen, T.D. and B.L. Boyce, An inverse finite element method for determining the anisotropic properties of the cornea. Biomech Model Mechanobiol, 2011. 10(3): p. 323-37.

42. Pandolfi, A. and G.A. Holzapfel, Three-dimensional modeling and computational analysis of the human cornea considering distributed collagen fibril orientations. J Biomech Eng, 2008. 130(6): p. 061006. 
43. Pandolfi, A. and F. Manganiello, A model for the human cornea: constitutive formulation and numerical analysis. Biomechanics and Modeling in Mechanobiology, 2006. 5(4): p. 237-246.

44. Avril, S., P. Badel, and A. Duprey, Anisotropic and hyperelastic identification of in vitro human arteries from full-field optical measurements. J Biomech, 2010. 43(15): p. 2978-85.

45. Fu, J., F. Pierron, and P.D. Ruiz, Elastic stiffness characterization using three-dimensional fullfield deformation obtained with optical coherence tomography and digital volume correlation. J Biomed Opt, 2013. 18(12): p. 121512.

46. Kim, J.-H., et al., Experimental characterization of rupture in human aortic aneurysms using a full-field measurement technique. Biomechanics and Modeling in Mechanobiology, 2012. 11(6): p. 841853.

47. Pierron, F. and M. Grediac, The virtual fields method: Extracting constitutive mechanical parameters from full-field deformation measurements. The Virtual Fields Method: Extracting Constitutive Mechanical Parameters from Full-field Deformation Measurements. 2012. 1-517.

48. Moussawi, A., et al., The constitutive compatibility method for identification of material parameters based on full-field measurements. Computer Methods in Applied Mechanics and Engineering, 2013. 265(0): p. 1-14.

49. Chakraborty, S. and P.D. Ruiz, Measurement of all orthogonal components of displacement in the volume of scattering materials using wavelength scanning interferometry. J Opt Soc Am A Opt Image Sci Vis, 2012. 29(9): p. 1776-85.

50. Bay, B.K., et al., Digital volume correlation: Three-dimensional strain mapping using X-ray tomography. Experimental Mechanics, 1999. 39(3): p. 217-226. 
51. Brault, R., et al., In-situ Analysis of Laminated Composite Materials by X-ray Micro-Computed Tomography and Digital Volume Correlation. Experimental Mechanics, 2013. 53(7): p. 1143-1151.

52. Gillard, F., et al., The application of digital volume correlation (DVC) to study the microstructural behaviour of trabecular bone during compression. Journal of the Mechanical Behavior of Biomedical Materials, 2014. 29(0): p. 480-499.

53. Limodin, N., et al., Influence of closure on the 3D propagation of fatigue cracks in a nodular cast iron investigated by X-ray tomography and 3D volume correlation. Acta Materialia, 2010. 58(8): p. 29572967.

54. Pierron, F., et al., Comparison of the Mechanical Behaviour of Standard and Auxetic Foams by X-ray Computed Tomography and Digital Volume Correlation. Strain, 2013. 49(6): p. 467-482.

55. Nahas, A., et al., 3D static elastography at the micrometer scale using Full Field OCT. Biomedical Optics Express, 2013. 4(10): p. 2138-2149.

56. Ortiz, S., et al., Optical coherence tomography for quantitative surface topography. Appl Opt, 2009. 48(35): p. 6708-15.

57. Podoleanu, A., et al., Correction of distortions in optical coherence tomography imaging of the eye. Physics in Medicine and Biology, 2004. 49(7): p. 1277-1294.

58. Van Der Jeught, S., et al., Real-time correction of geometric distortion artefacts in large-volume optical coherence tomography. Measurement Science and Technology, 2013. 24(5).

59. Patel, S., J. Marshall, and F.W. Fitzke Iii, Refractive index of the human corneal epithelium and stroma. Journal of Refractive Surgery, 1995. 11(2): p. 100-105. 
60. Westphal, V., et al., Correction of geometric and refractive image distortions in optical coherence tomography applying Fermat's principle. Optics Express, 2002. 10(9): p. 397-404.

61. Hamilton, K.E. and D.C. Pye, Young's modulus in normal corneas and the effect on applanation tonometry. Optometry and Vision Science, 2008. 85(6): p. 445-450.

62. MacRae, S., J. Schwiegerling, and R.W. Snyder, Customized and low spherical aberration corneal ablation design. J Refract Surg, 1999. 15(2 Suppl): p. S246-8.

63. Komai, Y. and T. Ushiki, The three-dimensional organization of collagen fibrils in the human cornea and sclera. Investigative Ophthalmology \& Visual Science, 1991. 32(8): p. 2244-2258. 